\title{
LAGRANGIANS AND HAMILTONIANS ON AFFINE BUNDLES AND HIGHER ORDER GEOMETRY
}

\author{
PAUL POPESCU and MARCELA POPESCU \\ Department of Applied Mathematics, University of Craiova \\ 13, Al. I. Cuza st., Craiova, 1100, Romania \\ E-mail: paul_popescu@k.ro
}

\begin{abstract}
The higher order bundles defined by an anchored bundle are constructed as a natural extension of the higher tangent spaces of a manifold. We prove that a hyperregular lagrangian (hyperregular affine hamiltonian) is a linearizable sub-lagrangian (affine sub-hamiltonian) on a suitable Legendre triple.
\end{abstract}

The anchored vector bundles are natural extensions of Lie algebroids, where the bracket misses. Usually, the anchor is supposed to be linear (as for example in [12], [2], [24]), but it can be also considered defined on affine bundles (as in [5], [16]); when it is used in control theory it can only be a bundle map ([3], [8]).

As already suggested in [10] in the case of Lie algebroids, the setting of anchored bundles allows to consider a lot of extensions of classical differential geometry. In this paper we are focused on higher order geometry, a central concept in Ehresman's work. We consider anchors defined on fibered manifolds. Even in the case of an affine bundle, the anchor is a bundle map, not necessary affine. The construction of higher order spaces is performed recursively. Anchored affine bundles of higher order are obtained starting from an anchored bundle and using Proposition 3.1. In the particular case of the tangent space, when the anchor is the identity, the higher order tangent spaces are obtained (studied, for example, in $[18,9])$.

The higher order tangent spaces, known as higher velocities bundles or bundles of accelerations, allow to consider higher order lagrangians and hamiltonians. The local equivalence problem of Euler and Hamilton equations can be considered in the hyperreg-

2000 Mathematics Subject Classification: Primary 55R10, 55R25, 53C60; Secondary 70H50, $22 \mathrm{~A} 30$.

Key words and phrases: affine bundle, anchored bundle, lagrangian, hamiltonian, Legendre map.

The authors are partially supported by the grant MEN-CNCSIS 81/2005.

The paper is in final form and no version of it will be published elsewhere. 
ular case (see Ostrogradski Theorem in [1, Chap. 3, Sect. 1.4]). The higher order tangent spaces are used in [9] by M. de Leon, performing a systematic study of mechanical systems. They are used also by R. Miron in [20, 18], in order to study higher order Finsler and Lagrange spaces. A dual theory of higher order Hamilton spaces was recently studied also by R. Miron in [21], using similar ideas of Ostrogradski's, but in a slight different way.

The affine hamiltonian considered in the paper is an affine section of a one dimensional affine bundle (see also [7]). A similar definition for special affine bundles is used in $[4,29]$. An affine hamiltonian allows to construct natural Legendre maps. In the case of a hyperregular lagrangian or an affine hamiltonian (i.e. when the Legendre map is a diffeomorphism), the Legendre map is called a Legendre transformation; it gives a one to one correspondence between hyperregular lagrangians and affine hamiltonians (see also [7]).

An affine section is used in [17]-[19] in order to construct a Legendre map. It makes the Legendre map as non-canonical associated to a lagrangian or a hamiltonian. The construction presented in our paper improves this aspect. In the case of a convex lagrangian, the Legendre transformation is considered in an equivalent but different way in [1, Chap. 3, Sect. 1.4]. The construction performed below is more general, since it works for all hyperregular lagrangians and hamiltonians of higher order. The Legendre map considered in $[11,30]$ for a lagrangian on a Lie algebroid has a natural extension to the higher order spaces, using the constructions performed in the paper.

A jet formalism for Lie algebroids is given in [15]. A higher order formalism for anchored bundles is proposed in our paper. One constructs recursively some higher order bundles $E^{k}$, which are affine bundles $E^{k} \rightarrow E^{k-1}$, where $k \geq 2$ and $E^{1}=E \rightarrow M$ is the initial anchored bundle. The hyperregular lagrangians and hamiltonians on $E^{k}$ are related one to one by means of a Legendre transformation between suitable bundles and they are viewed as linearizable sub-lagrangians and sub-hamiltonians on $E^{k-1}$.

Another goal of the paper is to introduce some ideas relevant to the study of singular lagrangians and hamiltonians. A minimal condition imposed in the paper on a singular lagrangian or hamiltonian is to allow a Legendre fibration on each fiber. An ingredient used in this context is a section of the Legendre fibration, called a Legendre section. We claim that this is not a restriction in many particular situations, the Legendre section coming from some geometric reasons. A Legendre section gives a Legendre triple, also defined in the paper. The linearization of a Legendre triple is also considered as a possibility to associate a lagrangian or a hamiltonian to a sub-lagrangian or a sub-hamiltonian of the triple, also defined in the paper.

In order to avoid complicated notations on fibers, we consider in the first section the cases of a vector space and an affine space respectively, giving detailed definitions and constructions. The case of affine bundles is studied in the second section. The higher order anchored bundles are defined in the third section, where the main result of the paper (Theorem 3.1) is proved.

1. Vectorial and affine hamiltonians and lagrangians. First we consider lagrangians and hamiltonians on a real finite dimensional vector space $V$. 
A lagrangian (a hamiltonian) on $V$ is a differentiable map $L: V \backslash V_{0} \rightarrow \mathbb{R}$ (respectively $H: V^{*} \backslash W_{0} \rightarrow \mathbb{R}$ ), where $V_{0} \subset V$ (respectively $W_{0} \subset V^{*}$ ) is a closed subset (for example an affine subspace). If the hessian of $L$ (respectively $H$ ) is non-degenerated in every point, then the Legendre map (i.e. the differential of $L$ or $H$ ) is a local diffeomorphism we say that $L$ or $H$ is regular. In particular, if the hessian of $L$ (respectively $H$ ) is strict positively defined, then $L$ (respectively $H$ ) is regular. If the Legendre map is a global diffeomorphism, then we say that $L$ (respectively $H$ ) is hyperregular and $\mathcal{L}$ is the Legendre transformation. In the hyperregular case, a lagrangian $L$ and a hamiltonian $H$ are related by $L\left(z^{i}\right)+H\left(\Omega_{i}\right)=z^{i} \Omega_{i}$.

Let us consider now lagrangians and hamiltonians on a real affine space $\mathcal{A}$, modelled on a real finite dimensional vector space $V$. The vectorial dual of $\mathcal{A}$ is $\mathcal{A}^{\dagger}=\operatorname{Aff}(\mathcal{A}, \mathbb{R})$, where $A f f$ denotes affine morphisms. There is a short exact sequence of vector spaces which has the form $0 \rightarrow \mathbb{R} \stackrel{j}{\rightarrow} \mathcal{A}^{\dagger} \stackrel{\pi}{\rightarrow} V^{*} \rightarrow 0$.

Let $\mathcal{R}=(o, \mathcal{B})$ and $\mathcal{R}^{\prime}=\left(o^{\prime}, \mathcal{B}^{\prime}\right)$ be two affine frames, where $o, o^{\prime} \in \mathcal{A}$ and $\mathcal{B}$, $\mathcal{B}^{\prime} \subset V$ are some bases. We denote by $\mathcal{B}^{*}=\left\{e^{i}\right\}_{i=\overline{1, m}} \subset V^{*}$ the dual base of $V^{*}$ and by $\mathcal{R}^{\dagger}=\left\{\tilde{e}^{0}, \tilde{e}^{i}\right\}_{i=1, m} \subset \mathcal{A}^{\dagger}$ the corresponding base, defined by $\tilde{e}^{0}=1$ and $\tilde{e}^{i}\left(E_{j}\right)=\delta_{j}^{i}$, where $e_{j}=\overrightarrow{o E_{j}}, \forall j=\overrightarrow{1, m}$. Considering the bases $\mathcal{R}^{\dagger},\left(\mathcal{R}^{\prime}\right)^{\dagger} \subset \mathcal{A}^{\dagger}$, then $\xi \in \mathcal{A}^{\dagger}$ has the forms $\xi=\omega \tilde{e}^{0}+\Omega_{i} \tilde{e}^{i}=\omega^{\prime} \tilde{e}^{0}+\Omega_{i^{\prime}} \tilde{e}^{i^{\prime}}$ and the following formulas hold:

$$
\Omega_{i^{\prime}}=a_{i^{\prime}}^{i} \Omega_{i}, \quad \omega^{\prime}=\omega+\Omega_{i} a^{i} .
$$

A lagrangian on $\mathcal{A}$ is a differentiable map $L: \mathcal{A} \backslash \mathcal{A}_{0} \rightarrow \mathbb{R}$, where $\mathcal{A}_{0} \subset \mathcal{A}$ is a closed subset (for example an affine subspace). If the hessian of $L$ is non-degenerate we say that $L$ is regular; in this case, the Legendre map $\mathcal{L}$ (i.e. the differential of $L$ ) is a local diffeomorphism. If the Legendre map is a global diffeomorphism on its image, then we say that $L$ is hyperregular and $\mathcal{L}$ is called the Legendre transformation. In particular, if the hessian of $L$ is strictly positive definite, then $L$ is hyperregular. The Legendre transformation $\mathcal{L}: \mathcal{A} \backslash \mathcal{A}_{0} \rightarrow V^{*}$ can relate a hyperregular lagrangian on $\mathcal{A}$ to a hyperregular hamiltonian on $V$ considering a point $z_{0} \in \mathcal{A} \backslash \mathcal{A}_{0}$ and using the relation $L\left(z^{i}\right)+H\left(\Omega_{i}\right)=\left(z^{i}-z_{0}^{i}\right) \Omega_{i}$, provided that $L$ or $H$ is regular. The consideration of $z_{0}$ gives a $H$, but it is not the only one, as we see below. We call a hamiltonian on $V$ a vectorial hamiltonian on $\mathcal{A}$.

An affine hamiltonian on $\mathcal{A}$ is a differentiable map $h: V^{*} \backslash W_{0} \rightarrow \mathcal{A}^{\dagger}$, such that $\pi \circ h=$ $1_{V^{*} \backslash W_{0}}$, where $W_{0} \subset V$ is a closed subset (for example an affine subspace). It was also considered in [7]. Using an affine frame $(o, \mathcal{B})$, then $h$ has the form $h\left(\Omega_{i}\right)=\left(\Omega_{i}, H_{0}\left(\Omega_{i}\right)\right)$. If another affine frame $\left(o^{\prime}, \mathcal{B}^{\prime}\right)$ is considered, then $H_{0}^{\prime}\left(\Omega_{i^{\prime}}\right)=H_{0}\left(\Omega_{i}\right)+\Omega_{i} a^{i}$. It follows that

$$
\frac{\partial^{2} H_{0}^{\prime}}{\partial \Omega^{i^{\prime}} \partial \Omega^{j^{\prime}}}=a_{i^{\prime}}^{i} a_{j^{\prime}}^{j} \frac{\partial^{2} H_{0}}{\partial \Omega^{i} \partial \Omega^{j}},
$$

thus the local functions $H_{0}^{\prime}$ and $H_{0}$ have the same hessian (viewed as a tensor), which depend only on $h$. We call the hessian tensor of $H_{0}^{\prime}$ and $H_{0}$ the hessian of $h$ and we say that $h$ is regular if the hessian is non-degenerate.

Let $h: V^{*} \backslash W_{0} \rightarrow \mathcal{A}^{\dagger}$ be an affine hamiltonian and consider a point $z_{0} \in \mathcal{A}$. The fact that $H_{0}\left(\Omega_{i}\right)-\Omega_{i} z_{0}^{i}=H_{0}^{\prime}\left(\Omega_{i^{\prime}}\right)-\Omega_{i} \cdot\left(z_{0}^{i}+a^{i}\right)=H_{0}^{\prime}\left(\Omega_{i^{\prime}}\right)-\Omega_{i} a_{i^{\prime}}^{i} z_{0}^{i^{\prime}}=H_{0}^{\prime}\left(\Omega_{i^{\prime}}\right)-\Omega_{i^{\prime}} z_{0}^{i^{\prime}}$ implies that $H_{z_{0}}\left(\Omega_{i}\right)=H_{0}\left(\Omega_{i}\right)-\Omega_{i} z_{0}^{i}$ defines a vectorial hamiltonian. It is easy to see that 
the hessian tensors of $h$ and $H_{z_{0}}$ are the same. Thus the vectorial and affine hamiltonians are related by the following result.

Proposition 1.1. If $z_{0} \in \mathcal{A}$ is a given point and $W_{0} \subset V^{*}$ is a closed subset, then there is a one to one correspondence between affine hamiltonians and vectorial hamiltonians on $V^{*} \backslash W_{0}$.

Notice that the correspondence defined above depends on the given point $z_{0} \in \mathcal{A}$.

A given point $z_{0} \in \mathcal{A}$ and the canonical duality $\varphi: V \times V^{*} \rightarrow \mathbb{R}$, define the Liouville map $C_{z_{0}}: \mathcal{A} \times V^{*} \rightarrow \mathbb{R}$, given by the formula $C_{z_{0}}(z, \Omega)=\varphi\left(z-z_{0}, \Omega\right)$, where $z-z_{0}$ denotes the vector $\overline{z_{0} z}$.

1.1. Hyperregular lagrangians and hamiltonians defined on open subsets of vector and affine spaces. It is easy to see that an affine hamiltonian $h$ is regular iff the vectorial hamiltonian $H_{z_{0}}$ is regular. We say that $h$ is hyperregular if $H_{z_{0}}$ is hyperregular.

Proposition 1.2. Let $L: \mathcal{A} \backslash \mathcal{A}_{0} \rightarrow \mathbb{R}$ be a hyperregular lagrangian on the real affine space $\mathcal{A}$ and $\mathcal{L}: \mathcal{A} \backslash \mathcal{A}_{0} \rightarrow V^{*} \backslash W_{0}$ be the Legendre transformation. Then for every point $z_{0} \in \mathcal{A}$, the map $H: V^{*} \backslash W_{0} \rightarrow \mathbb{R}, H(\Omega)=C_{z_{0}}\left(\mathcal{L}^{-1}(\Omega), \Omega\right)-L\left(\mathcal{L}^{-1}(\Omega)\right)$ is a hyperregular hamiltonian on $V^{*} \backslash W_{0}$ and the hyperregular affine hamiltonian $h: V^{*} \backslash W_{0} \rightarrow \mathcal{A}^{\dagger}$ corresponding to the point $z_{0}$ (according to Proposition 1.1) does not depend on the point $z_{0}$, depending only on the lagrangian $L$.

Proof. Using coordinates, the link between $L$ and $H$ is $L\left(z^{i}\right)+H\left(\Omega_{i}\right)=\left(z^{i}-z_{0}^{i}\right) \Omega_{i}$, where $\left.\mathcal{L}^{-1}(\Omega)\right)=z^{i} \bar{e}_{i}$. It is easy to check (classical) that $H$ is a hamiltonian. The affine hamiltonian corresponding to the point $z_{0}$ according to Proposition 1.1 has the form $\left(\Omega_{i}\right) \stackrel{h}{\rightarrow}$ $\left(\Omega_{i}, H_{0}\left(\Omega_{i}\right)\right)$, where $H_{0}\left(\Omega_{i}\right)=H\left(\Omega_{i}\right)+z_{0}^{i} \Omega_{i}=z^{i} \Omega_{i}-L\left(z^{i}\right)$, thus the conclusion follows.

A converse correspondence follows.

Proposition 1.3. Let $h: V^{*} \backslash W_{0} \rightarrow \mathcal{A}^{\dagger}$ be a hyperregular affine hamiltonian on the real affine space $\mathcal{A}$. Consider a point $z_{0} \in \mathcal{A}$, the hyperregular vectorial hamiltonian $H: V^{*} \backslash W_{0} \rightarrow \mathbb{R}$ corresponding to the point $z_{0}$ (according to Proposition 1.1), $\mathcal{H}:$ $V^{*} \backslash W_{0} \rightarrow V \backslash W_{1}$ its Legendre transformation and $\mathcal{A}_{0}=z_{0}+W_{1}$. Then

1. The map $\mathcal{H}_{0}: V^{*} \backslash W_{0} \rightarrow \mathcal{A} \backslash \mathcal{A}_{0}$ given by the formula $\mathcal{H}_{0}(\Omega)=\mathcal{H}(\Omega)+z_{0}$ is a diffeomorphism (called the Legendre transformation of $h$ ).

2. The real function $L: \mathcal{A} \backslash \mathcal{A}_{0} \rightarrow \mathbb{R}$ given by the formula $L(z)=C_{z_{0}}\left(z, \mathcal{H}^{-1}\left(z-z_{0}\right)\right)-$ $H\left(\mathcal{H}^{-1}\left(z-z_{0}\right)\right)$ is a hyperregular lagrangian.

3. Both $\mathcal{H}_{0}$ and $L$ do not depend on the point $z_{0}$, depending only on the affine hamiltonian $h$.

Proof. Using coordinates, $h$ has the form $\left(\Omega_{i}\right) \stackrel{h}{\rightarrow}\left(\Omega_{i}, H_{0}\left(\Omega_{i}\right)\right)$ and $H\left(\Omega_{i}\right)=H_{0}\left(\Omega_{i}\right)-$ $z_{0}^{i} \Omega_{i}$. Thus $\mathcal{H}(\Omega)^{i}=\partial H / \partial \Omega_{i}=\partial H_{0} / \partial \Omega_{i}-z_{0}^{i}$, then 1 . follows, since $h$ is regular. The proof of 2. uses a similar argument as in the lagrangian case. Using also coordinates, the link between $L$ and $H$ is also $L(z)+H(\Omega)=\left(z^{i}-z_{0}^{i}\right) \Omega_{i}$, where $\Omega=\Omega_{i} \bar{e}^{i}=\mathcal{H}^{-1}\left(z-z_{0}\right)$. It is also easy to check (classical) that $L$ is a lagrangian. If the affine hamiltonian $h$ has the form $h(\Omega)=\left(\Omega_{i} \bar{e}^{i}, H_{0}\left(\Omega_{i}\right)\right)$, then $H$ has the form $H(\Omega)=H_{0}(\Omega)-z_{0}^{i} \Omega_{i}$, where $\mathcal{H}^{-1}\left(z-z_{0}\right)=\Omega_{i} \bar{e}^{i}=\Omega$. Thus $L(z)=\left(z^{i}-z_{0}^{i}\right) \Omega_{i}-H(\Omega)=\left(z^{i}-z_{0}^{i}\right) \Omega_{i}-H_{0}(\Omega)+z_{0}^{i} \Omega_{i}=$ 
$z^{i} \Omega_{i}+H_{0}(\Omega)$, thus 2 . follows. Using coordinates, from the proof of 1 . it follows that the affine coordinates of $\mathcal{H}_{0}(\Omega)$ are $\left(\partial H_{0} / \partial \Omega_{i}\right)$, thus $\mathcal{H}_{0}$ depend only on $H_{0}$ and implicitly on $h$. Taking the coordinates $\left(z^{i}\right)$ of $z \in \mathcal{A} \backslash \mathcal{A}_{0}$ of the form $z^{i}=\partial H_{0} / \partial \Omega_{i}$ and denoting, as before, $\mathcal{H}^{-1}\left(z-z_{0}\right)=\Omega_{i} \bar{e}^{i}=\Omega$, we have $\mathcal{H}(\Omega)=z-z_{0}$. Using also 2 , we have $\mathcal{H}_{0}(\Omega)=\mathcal{H}(\Omega)+z_{0}=z$, thus $\Omega=\mathcal{H}_{0}^{-1}(z)$. Since $L(z)=z^{i} \Omega_{i}+H_{0}(\Omega)$, the conclusion follows.

1.2. Legendre triples and singular lagrangians and hamiltonians defined on subsets of vector and affine spaces. First we deal with the case of a (finite dimensional) vector space $V$. If $W \subset V$ is a vector subspace, we denote by $W^{0}=\left\{\omega \in V^{*} \mid \omega(v)=0\right.$, $\forall v \in W\}$, the polar of $W$. Analogously, if $W \subset V^{*}$ is a vector subspace, we denote by $W^{0}=\{v \in V \mid \omega(v)=0, \forall \omega \in W\}$, called also the polar of $W$.

A Legendre triple on $V$ is a triple $(X, \varphi, Y)$, where $X \subset V$ and $Y \subset V^{*}$ are closed submanifolds and $\varphi: X \rightarrow Y$ is a diffeomorphism. There are two affine bundles:

$\xi_{X}$ with base $X$, projection $\pi_{X}$ and fibers the affine subspaces $x+\left(T_{\varphi(x)} Y\right)^{0} \subset V$, $\forall x \in X$ and

$\eta_{Y}$ with base $Y$, projection $p_{Y}$ and fibers the affine subspaces $\varphi(y)+\left(T_{\varphi^{-1}(y)} X\right)^{0} \subset V^{*}$, $\forall y \in Y$.

The null sections of these affine bundles are defined by the natural inclusions $X \subset \xi_{X}$ and $Y \subset \eta_{Y}$.

We say that the Legendre triple is $V$-linearizable $\left(V^{*}\right.$-linearizable) if there is an open neighborhood $A_{X} \subset \xi_{X}$ (respectively $B_{Y} \subset \eta_{Y}$ ) of the null section such that $A_{X} \subset V$ (respectively $B_{Y} \subset V^{*}$ ) also as an open set. We say that the Legendre triple is linearizable if it is both $V$ and $V^{*}$ linearizable.

A sub-lagrangian (a sub-hamiltonian) on $V$ defined by the triple is a differentiable map $L: X \rightarrow \mathbb{R}$ (respectively $H: Y \rightarrow \mathbb{R}$ ). A sub-lagrangian $L$ and a sub-hamiltonian $H$ can be related by $L(y)+H(\varphi(v))=\omega(\varphi(v)), \forall v \in X$, the correspondence $L \leftrightarrow H$ is a bijection.

If the Legendre triple is $V^{*}$-linearizable and $L: X \rightarrow \mathbb{R}$ is a sub-lagrangian, then the formula

$$
H^{\prime}(\omega)=\omega\left(\varphi^{-1}\left(p_{Y}(\omega)\right)\right)-L\left(\varphi^{-1}\left(p_{Y}(\omega)\right)\right)
$$

gives a hamiltonian $H^{\prime}: B_{Y} \rightarrow \mathbb{R}$.

In an analogous way, if we assume that the Legendre triple is $V$-linearizable and $H: Y \rightarrow \mathbb{R}$ is a sub-hamiltonian, then the formula

$$
L^{\prime}(v)=\varphi\left(\pi_{X}(v)\right)(v)-H\left(\varphi\left(\pi_{X}(v)\right)\right)
$$

gives a lagrangian $L^{\prime}: A_{X} \rightarrow \mathbb{R}$.

A lagrangian $L: V \backslash V_{0} \rightarrow \mathbb{R}$ is singular if it is not regular. All the singular lagrangians considered in the paper have the image of the Legendre map $\mathcal{L}\left(V \backslash V_{0}\right) \subset V^{*}$ as a closed submanifold of $V^{*}$ and the Legendre map $\mathcal{L}$ is a submersion onto its image, defining the Legendre fibered manifold (L.f.m.) $\mathcal{L}: V \backslash V_{0} \rightarrow \mathcal{L}\left(V \backslash V_{0}\right)$; a section $s: \mathcal{L}\left(V \backslash V_{0}\right) \rightarrow V \backslash V_{0}$ of this fibered manifold (i.e. $\mathcal{L} \circ s=1_{\mathcal{L}\left(V \backslash V_{0}\right)}$ ) is called a Legendre section. The image $W=s\left(\mathcal{L}\left(V \backslash V_{0}\right)\right)$ is a closed submanifold of $V \backslash V_{0}$ that is transverse to the vertical fibers 
of the L.f.m. and $\mathcal{L}_{\mid W}: W \rightarrow \mathcal{L}\left(V \backslash V_{0}\right)$ is a diffeomorphism. Conversely, if $W \subset V \backslash V_{0}$ is a closed submanifold of $V \backslash V_{0}$ that is transverse to the vertical fibers of the L.f.m., intersecting each fiber in only one point, then $\mathcal{L}_{\mid W}: W \rightarrow \mathcal{L}\left(V \backslash V_{0}\right)$ is a diffeomorphism and its inverse defines a section $s$ of L.f.m. Thus a Legendre section of a singular lagrangian defines a Legendre triple. The restriction of $L$ to $W$ is a sub-lagrangian of this triple; if the triple is $V^{*}$-linearizable, then it defines a suitable hamiltonian.

EXAMPLE 0. Let us suppose that $\mathcal{L}\left(V \backslash V_{0}\right) \subset V^{*}$ is a closed hypersurface and there is a $c \in \mathbb{R}$ such that $L_{c}=\left\{z \in V \backslash V_{0}: L(z)=c\right\}$ is transverse to all the fibers of L.f.m. Thus there is a section of L.f.m. defined by $s=\mathcal{L}_{\mid L_{c}}^{-1}$, giving a Legendre triple.

ExAMPLE 1 . If $L: \mathbb{R}^{3} \backslash\{\overline{0}\} \rightarrow \mathbb{R}$ is defined by $L(x, y, z)=\sqrt{x^{2}+y^{2}+z^{2}}$, then

$$
\mathcal{L}(x, y, z)=\left(\frac{x}{\sqrt{x^{2}+y^{2}+z^{2}}}, \frac{y}{\sqrt{x^{2}+y^{2}+z^{2}}}, \frac{z}{\sqrt{x^{2}+y^{2}+z^{2}}}\right)
$$

and $\mathcal{L}\left(\mathbb{R}^{3} \backslash\{\overline{0}\}\right)=\left\{\left(p_{1}, p_{2}, p_{3}\right) \in \mathbb{R}^{3} ; p_{1}^{2}+p_{2}^{2}+p_{3}^{2}=1\right\} \cong S^{2}$. The vertical fibres of the Legendre map are all open rays that starts from the origin of $\mathbb{R}^{3}$. Every sphere $L_{c}$ defined by $L=c=$ const. $>0$ defines a Legendre section $s: \mathcal{L}\left(\mathbb{R}^{3} \backslash\{\overline{0}\}\right) \rightarrow L_{c}$, $\left(p_{1}, p_{2}, p_{3}\right) \rightarrow\left(c p_{1}, c p_{2}, c p_{3}\right)$ such that $\left(L_{c}, s, \mathcal{L}\left(\mathbb{R}^{3} \backslash\{\overline{0}\}\right)\right)$ is a linearizable Legendre triple. The restriction of $L$ to $L_{c}$ is a sub-lagrangian that defines the hamiltonian $H: \mathbb{R}^{3} \backslash\{\overline{0}\} \rightarrow$ $\mathbb{R}, H\left(p_{1}, p_{2}, p_{3}\right)=c \sqrt{p_{1}^{2}+p_{2}^{2}+p_{3}^{2}}-c$. Notice that in spite that $H_{\mid \mathcal{L}\left(\mathbb{R}^{3} \backslash\{\overline{0}\}\right)}=0$, this null sub-hamiltonian defines the lagrangian $L$.

EXAMPLE 2. A similar example can be considered for a lagrangian $L: \mathbb{R}^{3} \backslash N \rightarrow \mathbb{R}$ defined by $L(x, y, z)=\sqrt{x^{2}-y^{2}-z^{2}}$, where $N=\left\{(x, y, z) \in \mathbb{R}^{3} ; x^{2}-y^{2}-z^{2} \leq 0\right\}$. In this case $\mathcal{L}\left(\mathbb{R}^{3} \backslash N\right)=\left\{\left(p_{1}, p_{2}, p_{3}\right) \in \mathbb{R}^{3} ; p_{1}^{2}-p_{2}^{2}-p_{3}^{2}=1\right\}$. The vertical fibres of the Legendre map are the open rays that start from the origin and are not in $P$. The hyperboloid $L_{c}$ defined by $L=c=$ const. $>0$ defines also a Legendre section using the same formula. The restriction of $L$ to $L_{c}$ is a sub-lagrangian that defines the hamiltonian $H: \mathbb{R}^{3} \backslash N \rightarrow \mathbb{R}$, $H\left(p_{1}, p_{2}, p_{3}\right)=c \sqrt{p_{1}^{2}-p_{2}^{2}-p_{3}^{2}}-c$. As in the previous example, the null sub-hamiltonian (result from the restriction to $\mathcal{L}\left(\mathbb{R}^{3} \backslash N\right)$ ) defines the lagrangian $L$.

EXAMPLE 3. The examples can be generalized considering an Ehresmann connection on the L.f.m. of a singular lagrangian, such that the horizontal bundle of the connection is integrable, defining a horizontal foliation such that the restriction of the projection $\mathcal{L}$ is a diffeomorphism. Then, for every leaf of the horizontal foliation there is a Legendre section that has this leaf as its image.

Proposition 1.4. Let $L$ be a singular lagrangian, s be a section of L.f.m. such that the Legendre triple is $V^{*}$-linearizable. If $H$ is the corresponding hamiltonian and $\mathcal{H}$ is its Legendre* map, then:

1. The section $s$ is the restriction of $\mathcal{H}$ to $\mathcal{L}\left(V \backslash V_{0}\right)$, i.e. $s=\mathcal{H}_{\mid \mathcal{L}\left(V \backslash V_{0}\right)}$.

2. The image of $\mathcal{H}$ is $\mathcal{H}\left(A_{0}\right)=s\left(\mathcal{L}\left(V \backslash V_{0}\right)\right)$.

Proof. Let us consider some coordinates $\left(\Omega_{i}\right)_{i=\overline{1, n}}$ on $V^{*}$ such that it is possible to take $\left(\Omega_{\alpha}\right)_{\alpha=\overline{1, k}}$ as local coordinates on $\mathcal{L}\left(V \backslash V_{0}\right), k \leq n$. Thus a local parametrization on $\mathcal{L}\left(V \backslash V_{0}\right)$ is $\left(\Omega_{\alpha}\right) \rightarrow\left(\Omega_{\alpha}, f_{\bar{\alpha}}\left(\Omega_{\alpha}\right)\right)_{\alpha=\overline{1, k}, \bar{\alpha}=\overline{1, n-k}}$. 
Let us denote the local forms of $s, \pi_{0}$ and $H$ by

$$
\begin{gathered}
\left(\Omega_{i}\right) \stackrel{s}{\rightarrow}\left(s^{j}\left(\Omega_{i}\right)\right), \quad\left(\Omega_{i}\right) \stackrel{\pi_{0}}{\rightarrow}\left(\Pi_{\alpha}\left(\Omega_{i}\right), f_{\bar{\alpha}}\left(\Omega_{i}\right)\right) \quad \text { and } \\
\left(\Omega_{i}\right) \stackrel{H}{\rightarrow} \Omega_{j} s^{j}\left(\Pi_{\alpha}\left(\Omega_{i}\right), f_{\bar{\alpha}}\left(\Omega_{i}\right)\right)-L\left(s^{j}\left(\Pi_{\alpha}\left(\Omega_{i}\right), f_{\bar{\alpha}}\left(\Omega_{i}\right)\right)\right)
\end{gathered}
$$

respectively. Thus

$$
\begin{aligned}
\frac{\partial H}{\partial \Omega_{i}}= & s^{i}\left(\Pi_{\beta}\left(\Omega_{j}\right), f_{\bar{\beta}}\left(\Omega_{j}\right)\right)+\Omega_{j} \frac{\partial s^{j}}{\partial \Omega_{\beta}} \frac{\partial \Pi_{\beta}}{\partial \Omega_{i}}+\Omega_{j} \frac{\partial s^{j}}{\partial \Omega_{\bar{\beta}}} \frac{\partial f_{\bar{\beta}}}{\partial \Omega_{i}} \\
& -\frac{\partial L}{\partial y^{j}} \frac{\partial s^{j}}{\partial \Omega_{\beta}} \frac{\partial \Pi_{\beta}}{\partial \Omega_{i}}-\frac{\partial L}{\partial y^{j}} \frac{\partial s^{j}}{\partial \Omega_{\bar{\beta}}} \frac{\partial f_{\bar{\beta}}}{\partial \Omega_{i}} \\
= & s^{i}\left(\Pi_{\beta}\left(\Omega_{j}\right), f_{\bar{\beta}}\left(\Omega_{j}\right)\right),
\end{aligned}
$$

since using the construction of $\pi_{0}$ we have

$$
\left(\Omega_{j}-\frac{\partial L}{\partial y^{j}}\right) \frac{\partial s^{j}}{\partial \Omega_{\beta}}\left(\Pi_{\beta}\left(\Omega_{j}\right), f_{\bar{\beta}}\left(\Omega_{j}\right)\right)=\left(\Omega_{j}-\frac{\partial L}{\partial y^{j}}\right) \frac{\partial s^{j}}{\partial \Omega_{\bar{\beta}}}\left(\Pi_{\beta}\left(\Omega_{j}\right), f_{\bar{\beta}}\left(\Omega_{j}\right)\right)=0 .
$$

Then both assertions follow.

Since a hamiltonian on $V$ is a lagrangian on $V^{*}$, similar considerations are possible for a singular hamiltonian.

Let us consider a real affine space $\mathcal{A}$ modeled on a real and finite dimensional vector space $V$.

A Legendre triple on $\mathcal{A}$ is a triple $(X, \varphi, Y)$, where $X \subset \mathcal{A}$ and $Y \subset V^{*}$ are closed submanifolds and $\varphi: X \rightarrow Y$ is a diffeomorphism. As in the previous case, there are two affine bundles:

$\xi_{X}$ with base $X$, projection $\pi_{X}$ and as fibers the affine subspaces $\left(T_{\varphi(x)} Y\right)^{0}+x \subset \mathcal{A}$, $\forall x \in X$ and

$\eta_{Y}$ with base $Y$, projection $p_{Y}$ and as fibers the subspaces $\left(T_{\varphi^{-1}(y)} Y\right)^{0}+y \subset V^{*}$, $\forall y \in Y$.

The null sections of these affine bundles are defined by the natural inclusions $X \subset \xi_{X}$ and $Y \subset \eta_{Y}$.

We say that the Legendre triple is $\mathcal{A}$-linearizable $\left(V^{*}\right.$-linearizable) if there are open neighborhoods $A_{X} \subset \xi_{X}$ (respectively $B_{Y} \subset \eta_{Y}$ ) of the null section such that $A_{X} \subset \mathcal{A}$ (respectively $B_{Y} \subset V^{*}$ ) is also an open set. We say that the Legendre triple is linearizable if it is both $\mathcal{A}$ and $V^{*}$ linearizable.

A sub-lagrangian on $\mathcal{A}$ defined by the triple is a differentiable map $L: X \rightarrow \mathbb{R}$.

If the Legendre triple is $V^{*}$-linearizable, then a sub-lagrangian $L: X \rightarrow \mathbb{R}$ defines an affine hamiltonian $h^{\prime}: B_{Y} \rightarrow \mathcal{A}^{\dagger}$, as follows.

Proposition 1.5. Let us suppose that the Legendre triple is $V^{*}$-linearizable and let $L$ : $X \rightarrow \mathbb{R}$ be a sub-lagrangian. Then for every point $z_{0} \in \mathcal{A}$, the map $H_{z_{0}}: B_{Y} \rightarrow \mathbb{R}$, $H_{z_{0}}(\Omega)=C_{z_{0}}\left(\varphi^{-1} \circ p_{Y}(\Omega), \Omega\right)-L\left(\varphi^{-1} \circ p_{Y}(\Omega)\right)$ is a vectorial hamiltonian on $B_{Y}$ and the affine hamiltonian $h: B_{Y} \rightarrow \mathcal{A}^{\dagger}$ corresponding to the point $z_{0}$ (according to Proposition 1.1) does not depend on the point $z_{0}$, depending only on $L$ and $\varphi$. 
Proof. We can use similar arguments as in the proof of Proposition 1.2, where we consider $\varphi$ instead of $\mathcal{L}$, thus the coordinates $\left(z^{i}\right)$ are defined by $\varphi^{-1}(\Omega)=z^{i} \bar{e}_{i}$.

As in the case of a vector space, if a lagrangian $L: \mathcal{A} \backslash \mathcal{A}_{0} \rightarrow \mathbb{R}$ is not regular, it is singular. A minimal regularity condition is that the image of the Legendre map $\mathcal{L}\left(\mathcal{A} \backslash \mathcal{A}_{0}\right) \subset V^{*}$ is a closed submanifold of $V^{*}$ and the Legendre map $\mathcal{L}$ is a submersion onto its image, defining the Legendre fibered manifold (L.f.m.) $\mathcal{L}: \mathcal{A} \backslash \mathcal{A}_{0} \rightarrow \mathcal{L}\left(\mathcal{A} \backslash \mathcal{A}_{0}\right)$; a section $s: \mathcal{L}\left(\mathcal{A} \backslash \mathcal{A}_{0}\right) \rightarrow \mathcal{A} \backslash \mathcal{A}_{0}$ of this fibered manifold is called a Legendre section; it follows that $\mathcal{L} \circ s=1_{\mathcal{L}\left(V \backslash V_{0}\right)}$. The image $W=s\left(\mathcal{L}\left(\mathcal{A} \backslash \mathcal{A}_{0}\right)\right)$ is a closed submanifold of $\mathcal{A} \backslash \mathcal{A}_{0}$ that is transverse to the vertical fibers of the L.f.m. and $\mathcal{L}_{\mid W}: W \rightarrow \mathcal{L}\left(\mathcal{A} \backslash \mathcal{A}_{0}\right)$ is a diffeomorphism. Thus $\left(W, \mathcal{L}_{\mid W}, \mathcal{L}\left(V \backslash V_{0}\right)\right)$ is a Legendre triple; we say that it is the Legendre triple of $s$. On the other hand, considering a closed submanifold $W \subset \mathcal{A} \backslash \mathcal{A}_{0}$ that is transverse to the vertical fibers of the L.f.m., intersecting each fiber in only one point, then $\mathcal{L}_{\mid W}: W \rightarrow \mathcal{L}\left(V \backslash V_{0}\right)$ is a diffeomorphism and its inverse define a section $s$ of L.f.m., thus $\left(W, \mathcal{L}_{\mid W}, \mathcal{L}\left(V \backslash V_{0}\right)\right)$ is a Legendre triple; we say that it is the Legendre triple of $W$.

COROLlary 1.1. Let $L: \mathcal{A} \backslash \mathcal{A}_{0} \rightarrow \mathbb{R}$ be a singular lagrangian on the real affine space $\mathcal{A}, \mathcal{L}: \mathcal{A} \backslash \mathcal{A}_{0} \rightarrow \mathcal{L}\left(\mathcal{A} \backslash \mathcal{A}_{0}\right) \subset V^{*} \backslash W_{0}$ be its Legendre map and $s: \mathcal{L}\left(\mathcal{A} \backslash \mathcal{A}_{0}\right) \rightarrow \mathcal{A} \backslash \mathcal{A}_{0}$ be a Legendre section. We suppose that the Legendre triple of $s$ is $V^{*}$-linearizable. Then for every point $z_{0} \in \mathcal{A}$, the map $H: V^{*} \backslash W_{0} \rightarrow \mathbb{R}, H(\Omega)=C_{z_{0}}\left(s \circ \pi_{0}(\Omega), \Omega\right)-L(s \circ$ $\left.\pi_{0}(\Omega)\right)$ is a vectorial hamiltonian on $V^{*} \backslash W_{0}$ and the affine hamiltonian $h: V^{*} \backslash W_{0} \rightarrow \mathcal{A}^{\dagger}$ corresponding to the point $z_{0}$ (according to Proposition 1.1) does not depend on the point $z_{0}$, depending only on the lagrangian $L$ and the section $s$.

An affine sub-hamiltonian defined by a triple is a differentiable map $h: Y \rightarrow \mathcal{A}^{\dagger}$, such that $\pi \circ h=1_{Y}$; it can be regarded as a section of the restriction to $Y$ of the affine bundle $\mathcal{A}^{\dagger} \stackrel{\pi}{\rightarrow} V^{*}$. Since $Y \subset V^{*}$ a closed subset, it is possible to extend $h$ to a global section, i.e. an affine hamiltonian $h^{\prime}: V^{*} \rightarrow \mathcal{A}^{\dagger}$. Let $z_{0} \in \mathcal{A}$ be arbitrary taken and $H_{z_{0}}^{\prime}: V^{*} \rightarrow \mathbb{R}$ be the vectorial hamiltonian corresponding to the point $z_{0}$ according to Proposition 1.1. Then the restriction of $H_{z_{0}}^{\prime}$ to $Y$, denoted by $H_{z_{0}}: Y \rightarrow \mathbb{R}$, depends only on $z_{0}$ and $h$, it does not depend on the extension $h^{\prime}$.

Lemma 1.1. Let us suppose that the Legendre triple is $\mathcal{A}$-linearizable. Then the map $E: A_{X} \times Y \rightarrow \mathbb{R}, E(z, \Omega)=C_{z_{0}}(z, \Omega)-H_{z_{0}}(\Omega)$ does not depend on $z_{0}$, it depends only on $h$.

Proof. Using coordinates, denote by $\left(z^{i}\right),\left(\Omega_{i}\right)$ and $\left(z_{0}^{i}\right)$ the coordinates of $z, \Omega$ and $z_{0}$ respectively. Then $E$ has the local form $\left(z^{i}, \Omega_{j}\right) \rightarrow\left(z^{i}-z_{0}^{i}\right) \Omega_{i}-\left(H_{0}(\Omega)-z_{0}^{j} \Omega_{j}\right)=$ $z^{j} \Omega_{j}-H_{0}(\Omega)$.

We define a lagrangian $L: A_{0} \rightarrow \mathbb{R}$ using the formula

$$
L(z)=C_{z_{0}}\left(z, \varphi \circ \pi_{X}(z)\right)-H_{z_{0}}\left(\varphi \circ \pi_{X}(z)\right) .
$$

Using Lemma 1.1 we can prove the following result.

Proposition 1.6. Let us suppose that the Legendre triple is $\mathcal{A}$-linearizable. Then the lagrangian $L: B \rightarrow \mathbb{R}$ does not depend on $z_{0}$, it depends only on $h$ and $s$. 
We are going to prove similar results as stated in Proposition 1.3.

Let $h: V^{*} \backslash W_{0} \rightarrow \mathcal{A}^{\dagger}$ be an affine hamiltonian on $\mathcal{A}$. Consider a point $z_{0} \in \mathcal{A}$, the vectorial hamiltonian $H: V^{*} \backslash W_{0} \rightarrow \mathbb{R}$ corresponding to the point $z_{0}$ (according to Proposition 1.1), the Legendre map $\mathcal{H}: V^{*} \backslash W_{0} \rightarrow V \backslash W_{1}$ and $\mathcal{A}_{0}=z_{0}+W_{1}$. Consider the map $\mathcal{H}_{0}: V^{*} \backslash W_{0} \rightarrow \mathcal{A} \backslash \mathcal{A}_{0}$ given by the formula $\mathcal{H}_{0}(\Omega)=\mathcal{H}(\Omega)+z_{0}$ (called the Legendre map of $h)$. Using coordinates, $h$ has the form $\left(\Omega_{i}\right) \stackrel{h}{\rightarrow}\left(\Omega_{i}, H_{0}\left(\Omega_{i}\right)\right)$ and $H\left(\Omega_{i}\right)=$ $H_{0}\left(\Omega_{i}\right)-z_{0}^{i} \Omega_{i}$. It follows that $\mathcal{H}(\Omega)^{i}=\partial H / \partial \Omega_{i}=\partial H_{0} / \partial \Omega_{i}-z_{0}^{i}$ and $\mathcal{H}_{0}(\Omega)^{i}=\partial H_{0} / \partial \Omega_{i}$, thus $\mathcal{H}_{0}$ depends only on $h$.

We suppose that $\mathcal{H}_{0}\left(V^{*} \backslash W_{0}\right)$ is a closed submanifold of $\mathcal{A}$ and $\mathcal{H}_{0}: V^{*} \backslash W_{0} \rightarrow$ $\mathcal{H}_{0}\left(V^{*} \backslash W_{0}\right)$ is a fibered manifold, called the Legendre* fibered manifold (L*.f.m.) of $h$. We suppose that a section $s: \mathcal{H}_{0}\left(V^{*} \backslash W_{0}\right) \rightarrow V^{*} \backslash W_{0}$ of the $\mathrm{L}^{*}$..f.m. is given, called a Legendre ${ }^{*}$ section. Denoting $W=s\left(\mathcal{H}_{0}\left(V^{*} \backslash W_{0}\right)\right)$, then $s: \mathcal{H}_{0}\left(V^{*} \backslash W_{0}\right) \rightarrow W$ is a diffeomorphism, thus $\left(\mathcal{H}_{0}\left(V^{*} \backslash W_{0}\right), s, W\right)$ is a Legendre triple; we say that it is the Legendre triple of $s$ (or $W$ ).

Also, if $W \subset V^{*} \backslash W_{0}$ is a closed submanifold that is transverse to the vertical fibers of the $\mathrm{L}^{*}$.f.m., intersecting each fiber in only one point, then $\mathcal{H}_{0 \mid W}: W \rightarrow \mathcal{H}_{0}\left(V^{*} \backslash W_{0}\right)$ is a diffeomorphism and its inverse define a section $s$ of $\mathrm{L}^{*}$.f.m., thus $\left(\mathcal{H}_{0}\left(V^{*} \backslash W_{0}\right), \mathcal{H}_{0 \mid W}^{-1}, W\right)$ is a Legendre triple; we say that it is the Legendre triple of $s$ (or $W$ ).

COROLlary 1.2. Let us suppose that the Legendre triple defined by a Legendre* section $s$ is $\mathcal{A}$-linearizable. Then there is a lagrangian that depends only on $h$ and $s$.

Notice that analogous results as stated in Proposition 1.4 can be proved in the affine case, but we do not need them in that follows.

2. Lagrangians and affine hamiltonians on affine bundles. In this section we study lagrangians and affine hamiltonians on affine bundles in both hyperregular and singular cases.

The hyperregular lagrangians and hyperregular affine hamiltonians are canonically related by Legendre and Legendre* transformations in a canonical way. In particular, a lagrangian on an affine bundle and an affine section defines a vectorial hamiltonian. This correspondence lagrangian-vectorial hamiltonian is not canonical, depending on the section; it is the situation essentially used in $[18,19]$.

In order to study singular lagrangians and singular affine hamiltonians, we define Legendre triples, sub-lagrangians and (affine) sub-hamiltonians. We consider also a Legendre triple on each fiber, defined by a section of the Legendre fibered manifold.

In that follows $E \stackrel{\pi}{\rightarrow} M$ is an affine bundle and $\bar{E} \stackrel{\bar{\pi}}{\rightarrow} M$ is the corresponding vector bundle. We consider also an open fibered submanifold $\tilde{E} \stackrel{\hat{\pi}}{\rightarrow} M$ of the affine bundle (i.e. its fibers are open submanifolds of the affine fibers). If $L: \tilde{E} \rightarrow \mathbb{R}$ is differentiable, we say that $L$ is a lagrangian on $E$.

An interesting example is the affine bundle $T^{k} M \rightarrow T^{k-1} M, k \geq 1$, where $T^{k} M$ is the $k$-tangent space of a manifold $M$. Then $T^{1} M \rightarrow M$ is the tangent bundle, $T^{2} M \rightarrow M$ is the tangent bundle of order two etc. A lagrangian of order $k$ on $M$ is $L^{k}: T^{k} M \backslash s_{0}(M)$ $\rightarrow \mathbb{R}$, where $s_{0}: M \rightarrow T^{k} M$ is the null section. Lagrangians of order $k \geq 1$ that are continuous on $T^{k} M$ and smooth on $T^{k} M \backslash s_{0}(M)$ are considered in [18]. 
If $L: \tilde{E} \rightarrow \mathbb{R}$ is a lagrangian, then the Legendre maps on fibers induce the Legendre map, i.e. a fibered manifold map $\mathcal{L}: \tilde{E} \rightarrow \bar{E}^{*}$. Using local coordinates, we have $\left(x^{i}, y^{\alpha}\right) \stackrel{\mathcal{L}}{\rightarrow}$ $\left(x^{i},\left(\partial L / \partial y^{\beta}\right)\left(x^{i}, y^{\alpha}\right)\right)$. It is easy to see that if $L$ is a hyperregular lagrangian, then $\mathcal{L}$ is a global diffeomorphism.

The Legendre map defines an $\mathcal{L}$-morphism of the vertical vector bundles $V E \rightarrow V \bar{E}^{*}$ (called the vertical Legendre morphism) and expressed in local coordinates by

$$
\left(x^{i}, y^{\alpha}, Y^{\beta}\right) \rightarrow\left(x^{i}, \frac{\partial L}{\partial y^{\beta}}\left(x^{i}, y^{\alpha}\right), Y^{\beta} \frac{\partial^{2} L}{\partial y^{\beta} y^{\gamma}}\left(x^{i}, y^{\alpha}\right)\right) \text {. }
$$

Let $\check{E} \stackrel{\check{\pi}}{\rightarrow} M$ be an open fibered submanifold of the vector bundle $\bar{E} \stackrel{\bar{\pi}}{\rightarrow} M$. An affine hamiltonian on $E$ is a fibered manifold map $h: \check{E} \rightarrow E^{\dagger}$ that is an affine hamiltonian $h_{x}: \check{E}_{x} \rightarrow E_{x}^{\dagger}, \forall x \in M$, i.e. on each fiber.

2.1. Hyperregular lagrangians and hamiltonians. The lagrangian $L: \tilde{E} \rightarrow \mathbb{R}$ is hyperregular if it is hyperregular on each fiber.

THEOREM 2.1. Let $L: \tilde{E} \rightarrow \mathbb{R}$ be a hyperregular lagrangian. Then there is a hyperregular affine hamiltonian $h: \mathcal{L}(\tilde{E}) \rightarrow E^{\dagger}$ defined by $L$ such that the vertical Legendre morphism is an isometry.

Proof. Using Proposition 1.2, the affine hamiltonian $h$ has the local form $\left(p_{\alpha}\right) \stackrel{h}{\rightarrow}$ $\left(p_{\alpha}, H_{0}\left(p_{\alpha}\right)\right)$, where $H_{0}: \bar{E}^{*} \rightarrow \mathbb{R}$ is defined using the formula

$$
s H_{0}\left(x^{i}, p_{\alpha}\right)=p_{\alpha} H^{\alpha}\left(x^{i}, p_{\alpha}\right)-L\left(x^{i}, H^{\gamma}\left(x^{i}, p_{\alpha}\right)\right) .
$$

Here $\mathcal{H}$ is the inverse of the Legendre transformation, $\mathcal{H}=\mathcal{L}^{-1}$ and it has the local form $\mathcal{H}\left(x^{i}, p_{\alpha}\right)=\left(x^{i}, H^{\gamma}\left(x^{i}, p_{\alpha}\right)\right)$.

If $E \rightarrow M$ is an affine bundle, then the vector bundle $\bar{E} \rightarrow M$, canonically associated with $E$, which can be regarded as a central affine bundle. We denote by $\bar{E}^{*} \rightarrow M$ its dual vector bundle. A vectorial hamiltonian defined by $E$ is a lagrangian $H: \bar{E}^{*} \rightarrow \mathbb{R}$ defined on the central affine bundle $\bar{E}^{*}$. If $s: M \rightarrow E$ is an affine section, then using Proposition 1.1, one can construct a one to one correspondence between vectorial and affine hamiltonians on $E$.

Corollary 2.1. Let $s: M \rightarrow \tilde{E}$ be an affine section and $L: \tilde{E} \rightarrow \mathbb{R}$ a hyperregular lagrangian. Then there is a vectorial hamiltonian $H: \mathcal{L}(\tilde{E}) \rightarrow \mathbb{R}$ such that the vertical Legendre morphism is an isometry and the vertical hessian of $H$ does not depend on the section s.

In what follows $\check{E} \subset \bar{E}^{*}$ is an open fibered submanifold.

THEOREM 2.2. Let $h: \check{E} \rightarrow E^{\dagger}$ be a hyperregular affine hamiltonian. Then there is a hyperregular lagrangian $L: \tilde{E}=\mathcal{H}(\check{E}) \rightarrow \mathbb{R}$ such that the vertical Legendre* morphism is an isometry.

Proof. Using Proposition 1.3, the lagrangian $L$ has the form

$$
L\left(x^{i}, y^{\alpha}\right)=L_{\alpha}\left(x^{i}, y^{\alpha}\right) y^{\alpha}-H_{0}\left(x^{i}, L_{\gamma}\left(x^{i}, y^{\alpha}\right)\right),
$$


where the affine hamiltonian $h$ has the local form $\left(x^{i}, p_{\alpha}\right) \stackrel{h}{\rightarrow}\left(p_{\alpha}, H_{0}\left(x^{i}, p_{\alpha}\right)\right)$ and $\mathcal{L}=$ $\mathcal{H}^{-1}$, the inverse of the Legendre* transformation and given on each fiber by Proposition 1.3), has the local form $\left.\mathcal{L}\left(x^{i}, y^{\alpha}\right)=\left(x^{i}, L_{\gamma}\left(x^{i}, y^{\alpha}\right)\right)\right)$.

COROLlary 2.2. Let $s: M \rightarrow E$ be an affine section and $H: \check{E} \rightarrow \mathbb{R}$ be a hyperregular vectorial hamiltonian. Then there is a hyperregular lagrangian $L: \mathcal{H}(\check{E}) \rightarrow \mathbb{R}$ on $E$ such that the vertical Legendre* morphism is an isometry and the vertical hessian of $L$ does not depend on the section $s$.

Notice that the Legendre and Legendre* transformations are more natural in the case of an affine hamiltonian and do not depend on a section $s: M \rightarrow \tilde{E}$; in the case of a vectorial hamiltonian $H$ the Legendre and Legendre* transformations depend both on the section $s$.

2.2. Sub-lagrangians, sub-hamiltonians and singular lagrangians and hamiltonians. Let us consider an affine bundle $E \stackrel{\pi}{\rightarrow} M$ and $\bar{E} \stackrel{\bar{\pi}}{\rightarrow} M$ be its associated vector bundle. We say that $\left(E^{\prime}, \varphi, E^{\prime \prime}\right)$ is an affine Legendre triple if the following three conditions are fulfilled:

$E^{\prime} \stackrel{\pi^{\prime}}{\rightarrow} M$ is a fibered submanifold of $E$,

$E^{\prime \prime} \stackrel{\pi^{\prime \prime}}{\rightarrow} M$ is a fibered submanifold of $\bar{E}^{*} \stackrel{\bar{\pi}^{\prime}}{\rightarrow} M$ and

$\varphi: E^{\prime} \rightarrow E^{\prime \prime}$ is an isomorphism of fibered manifolds.

If $E \stackrel{\pi}{\rightarrow} M$ is a vector bundle, then $\left(E^{\prime}, \varphi, E^{\prime \prime}\right)$ is called a vectorial Legendre triple.

We say that the Legendre triple is E-linearizable ( $\bar{E}^{*}$-linearizable) if the Legendre triple induced on every fiber is respectively linearizable (i.e. the Legendre triple $\left(E_{x}^{\prime}, \varphi_{x}, E_{x}^{\prime \prime}\right)$ is $E_{x}$-linearizable, respectively $\bar{E}_{x}^{*}$-linearizable $\left.\forall x \in M\right)$. It is possible that there exists an open fibered submanifold $E_{0}^{\prime} \stackrel{\pi_{0}^{\prime}}{\rightarrow} M$ of $E \stackrel{\pi}{\rightarrow} M$ (respectively $E_{0}^{\prime \prime} \stackrel{\pi_{0}^{\prime \prime}}{\rightarrow} M$ of $\left.\bar{E}^{*} \stackrel{\bar{\pi}}{\rightarrow} M\right)$ such that on every fiber $\left(E_{0}^{\prime}\right)_{x}=A_{E_{x}^{\prime}} \subset \xi_{E_{x}^{\prime}}\left(\right.$ respectively $\left.\left(E_{0}^{\prime \prime}\right)_{x}=B_{E_{x}^{\prime \prime}} \subset \eta_{E_{x}^{\prime \prime}}\right)$ are also open subsets, $\forall x \in M$. (The notations are as in subsection 1.2.)

We say that the Legendre triple is E-linearizable if it is both $E$ - and $\bar{E}^{*}$-linearizable.

For a given Legendre triple $\left(E^{\prime}, \varphi, E^{\prime \prime}\right)$ :

A sub-lagrangian is a differentiable map $L: E^{\prime} \rightarrow \mathbb{R}$.

A vectorial sub-hamiltonian is a differentiable map $H: E^{\prime \prime} \rightarrow \mathbb{R}$.

An affine sub-hamiltonian is a differentiable map $h: E^{\prime \prime} \rightarrow E^{\dagger}$ that is an affine sub-hamiltonian $h_{x}: E_{x}^{\prime \prime} \rightarrow E_{x}^{\dagger}$ on each fiber, for every $x \in M$.

EXAMPLES (of Legendre triples). 1. Let $(M, g)$ be a riemannian manifold, and consider the canonical vector bundle isomorphism $g^{\#}: T M \rightarrow T^{*} M$; then the triple $\left(T M, g^{\#}, T^{*} M\right)$ is a vectorial Legendre triple. The quadratic forms $g^{\prime}(X)=g(X, X)$ and $g^{\prime \prime}(\omega)=g^{*}(\omega, \omega)$ (where $g^{*}$ is the dual of $g$ ) are a sub-lagrangian, respectively a vectorial sub-hamiltonian of this triple. Denoting by $S^{1} M \rightarrow M$ and $S^{* 1} M \rightarrow M$ the bundle of unit spheres $\left(S^{1} M \subset T M\right.$ and $\left.S^{* 1} M \subset T^{*} M\right)$, then the restriction $g_{1}^{\#}$ of $g^{\#}$ to $S^{1} M$ defines a Legendre triple $\left(S^{1} M, g_{1}^{\#}, S^{* 1} M\right)$. The restrictions of the quadratic forms $g^{\prime}$ and $g^{\prime \prime}$ to $S^{1} M$ and $S^{* 1} M$ respectively are a sub-lagrangian, respectively a vectorial sub-hamiltonian of this triple. 
2. Let $L: \widetilde{T M} \rightarrow \mathbb{R}$ be a hyperregular lagrangian such that $\mathcal{L}(\widetilde{T M})=\widetilde{T^{*} M}$; then $\left(\widetilde{T M}, \mathcal{L}, \widetilde{T^{*} M}\right)$ is a vectorial Legendre triple. Notice that we can consider the more general example of an open fibered submanifold $E_{0} \subset T M$; if $L: E_{0} \rightarrow \mathbb{R}$ is hyperregular, then $\left(E_{0}, \mathcal{L}, L\left(E_{0}\right)\right)$ is a vectorial Legendre triple.

3. If $L: \tilde{E} \rightarrow \mathbb{R}$ is a hyperregular lagrangian, then $(\tilde{E}, \mathcal{L}, \mathcal{L}(\tilde{E}))$ is a Legendre triple. We can regard $L$ as a sub-lagrangian and its dual affine hamiltonian $h$ can be regarded as well as an affine sub-hamiltonian.

4. Let $\tilde{E} \stackrel{\tilde{\pi}}{\rightarrow} M$ be an open fibered submanifold of an affine bundle $E \stackrel{\pi}{\rightarrow} M$ and $L: \tilde{E} \rightarrow \mathbb{R}$ be a lagrangian such that, $\forall x \in M, \mathcal{L}_{x}\left(\tilde{E}_{x}\right) \subset \bar{E}_{x}^{*}$ is a hypersurface and there is $c \in \mathbb{R}$ such that $L^{-1}(c) \cap \tilde{E}_{x}:=L_{x}^{-1}(c)$ is transverse to the fibers of the Legendre fibered manifold $\mathcal{L}_{x}: \tilde{E}_{x} \rightarrow \mathcal{L}_{x}\left(\tilde{E}_{x}\right)$. Then the restrictions $\varphi_{x}$, of $\mathcal{L}_{x}$ to $L_{x}^{-1}(c)$, define the affine Legendre triple $(\mathcal{L}, \varphi, \mathcal{L}(\tilde{E}))$. If $E \stackrel{\pi}{\rightarrow} M$ is a vector bundle, then $(\mathcal{L}, \varphi, \mathcal{L}(\tilde{E}))$ is a vectorial Legendre triple. The next example is a simple particular case.

5. We use the data from the first example and we consider $L=g_{1}^{\prime}=\sqrt{g^{\prime}}: \widetilde{T M} \rightarrow \mathbb{R}$, i.e. $g_{1}^{\prime}(X)=\sqrt{g(X, X)}$. Then $\mathcal{L}(\widetilde{T M})=S^{* 1} M$ and $S^{1} M=L_{1}^{-1}$ is transverse to the fibers of the Legendre fibered manifold on every fiber. Following Example 4 we recover the Legendre triple $\left(S^{1} M, g_{1}^{\#}, S^{* 1} M\right)$ already considered in the first example.

Proposition 2.1. If the Legendre triple is E-linearizable ( $\bar{E}^{*}$-linearizable) and $L: E^{\prime} \rightarrow$ $\mathbb{R}$ is a sub-lagrangian (respectively $h: E^{\prime \prime} \rightarrow E^{\dagger}$ is an affine sub-hamiltonian), then using Proposition 1.5 (respectively Proposition 1.6) on each fiber, one obtain an affine hamiltonian $h: E_{0}^{\prime \prime} \rightarrow E^{\dagger}$ (respectively a lagrangian $L: E_{0}^{\prime} \rightarrow \mathbb{R}$ ).

In the sequel, the singular lagrangians and hamiltonians induce, on each fiber, singular lagrangians and hamiltonians as studied in subsection 1.1.

Let $\tilde{E} \stackrel{\tilde{\pi}}{\rightarrow} M$ be an open fibered submanifold of an affine bundle $E \stackrel{\pi}{\rightarrow} M$. We say that a lagrangian $L: \tilde{E} \rightarrow \mathbb{R}$ is singular if there is a Legendre fibered manifold $\tilde{E}_{x} \rightarrow \mathcal{L}_{x}\left(\tilde{E}_{x}\right)$ on each fiber $\tilde{E}_{x}, \forall x \in M$, defining a (global) Legendre fibered manifold $\tilde{E} \rightarrow \mathcal{L}(\tilde{E})$. Additionally, a (global) Legendre section $s: \mathcal{L}(\tilde{E}) \rightarrow \tilde{E}$ is used, inducing a Legendre section $s_{x}: \mathcal{L}_{x}\left(\tilde{E}_{x}\right) \rightarrow \tilde{E}_{x}$ on each fiber. The Legendre section $s$ defines and is uniquely defined by a Legendre triple $(W, \mathcal{L} \mid W, \mathcal{L}(\tilde{E}))$, where $W=s(\mathcal{L}(\tilde{E}))$ is transverse to the fibers of the Legendre fibration.

We say that the lagrangian $L$ is linearizable* with respect to the Legendre section $s$ if the associated Legendre triple is $\bar{E}^{*}$-linearizable.

We follow a similar way using an affine hamiltonian. Let $\check{E} \stackrel{\tilde{\pi}}{\rightarrow} M$ be an open fibered submanifold of the vector bundle $\bar{E}^{*} \stackrel{\pi}{\rightarrow} M$. We say that an affine hamiltonian $h: \check{E} \rightarrow E^{\dagger}$ is singular if there is a Legendre fibered manifold $\check{E}_{x} \rightarrow \mathcal{H}_{x}\left(\check{E}_{x}\right)$ on each fiber $\check{E}_{x}, \forall x \in$ $M$, defining a (global) Legendre* fibered manifold $\check{E} \rightarrow \mathcal{H}(\check{E})$. Additionally, a (global) Legendre* section $s: \mathcal{H}(\check{E}) \rightarrow \check{E}$ is used, inducing a Legendre section $s_{x}: \mathcal{H}_{x}\left(\check{E}_{x}\right) \rightarrow \check{E}_{x}$ on each fiber. The Legendre* section $s$ defines and is uniquely defined by a Legendre triple $\left(\mathcal{H}(W),\left(\mathcal{H}_{\mid W}\right)^{-1}, W\right)$, where $W=s(\mathcal{H}(\check{E}))$ is transverse to the fibers of the Legendre* fibration.

We say that the affine hamiltonian $h$ is linearizable according to the Legendre* section $s$ if the associated Legendre triple is $E$-linearizable. 
COROLlary 2.3. If the lagrangian $L: \tilde{E} \rightarrow \mathbb{R}$ is linearizable* (the affine hamiltonian $h: \check{E} \rightarrow E^{\dagger}$ is linearizable) then using Proposition 1.5 (respectively using Proposition 1.6) on each fiber, we obtain an affine hamiltonian $h: E_{0}^{\prime \prime} \rightarrow E^{\dagger}$ (respectively a lagrangian $\left.L: E_{0}^{\prime} \rightarrow \mathbb{R}\right)$.

3. Higher order anchored bundles. An inductive definition of the higher order spaces $T^{k} M$ is given, for example, in [1, Chap. 3, Sect. 1.4], where the notation $J^{k}$ is used for $T^{k} M$. We briefly describe this construction. Using our notations, $T^{0} M=M, T^{1} M=$ $T M, \pi_{1}: T^{1} M \rightarrow T^{0} M$ is the canonical projection and for $k \geq 1, T^{k+1} M$ is the affine subbundle of the tangent bundle $T T^{k} M$ of vectors $\xi \in T_{x} T^{k} M$ such that considering the differential $\pi_{k *}: T_{x} T^{k} M \rightarrow T_{\pi(x)} T^{k-1} M$ of the projection $\pi_{k}: T^{k} M \rightarrow T^{k-1} M$, then $\pi_{k *}(\xi)=x$ and $\pi_{k+1}: T^{k+1} M \rightarrow T^{k} M$ is induced by the canonical projection $T T^{k} M \rightarrow T^{k} M$. Notice that there is an inclusion map $h_{k}: T^{k} M \rightarrow T T^{k-1} M$, which is an affine bundle map. The definition of $T^{k} M$ is very simple and has a geometric description. We extend this construction, starting from an anchored bundle and constructing higher anchored bundles. We prove that the hyperregular lagrangians and affine hamiltonians defined on these bundles define linearizable triples on suitable tangent bundles.

Consider a fibered manifold $E \stackrel{\pi}{\rightarrow} M$. An anchor on $E$ is a $\pi$-morphism $\rho: E \rightarrow T M$. It can be viewed also as a section $\Gamma: E \rightarrow \pi^{*} T M$ of the induced vector bundle $\pi^{*} T M$, where $T M \stackrel{p}{\rightarrow} M$ is the tangent bundle of $M$. Using local coordinates, $\left(x^{i}, y^{\alpha}\right) \stackrel{\rho}{\rightarrow}\left(x^{i}, \rho^{i}\left(x^{j}, y^{\alpha}\right)\right)$ and $\Gamma=\rho^{i}\left(x^{j}, y^{\alpha}\right) \partial / \partial x^{i}$.

1) Let $E \stackrel{\pi}{\rightarrow} M$ be a fibered manifold and $X \in \mathcal{X}(M)$ be a vector field on the base $M$. Then $X$ defines an anchor on $E$ by the formula $\rho(e)=X_{\pi(e)}$. Using local coordinates, $\left(x^{i}, y^{\alpha}\right) \stackrel{\rho}{\rightarrow}\left(x^{i}, X\left(x^{i}\right)\right)$ and $\Gamma=X\left(x^{i}\right) \partial / \partial x^{i}=X$.

2) The identity morphism of the tangent bundle defines a (canonical) anchor $\rho=$ $I: T M \rightarrow T M$. Using local coordinates, $\left(x^{i}, y^{j}\right) \stackrel{I}{\rightarrow}\left(x^{i}, y^{j}\right)$ and $\Gamma=y^{i} \partial / \partial x^{i}$. This example can be also interpreted using the Liouville vector field $\ell$ as follows. The vertical vector bundle $V T M \rightarrow T M$ is canonically isomorphic with the vector bundle $p^{*} T M$. Thus the Liouville vector field $\ell$ of $V T M$ corresponds to the canonical anchor $I$ of $T M$. Consider some local coordinates $\left(x^{i}, y^{j}\right)$ on $T M$, which correspond to an open set $U \subset M$, then the Liouville vector field has the local forms $\ell=y^{j} \partial / \partial y^{j}$ as a vertical section and $\Gamma=y^{j} \partial / \partial x^{j}$ as a section $\Gamma: T M \rightarrow p^{*} T M$.

4) Any endomorphism $F$ of the tangent bundle defines an anchor of the tangent bundle (for example: an almost complex structure: $F^{2}=-I$, an almost product structure $F^{2}=I$, or an almost tangent structure $F^{2}=0$ ).

5) The above example 3) can be extended. Let $\pi_{k}: T^{k} M \rightarrow T^{k-1} M$ be the affine bundle defined by the total space of the tangent bundle of order $k$. Then the inclusion $h_{k}: T^{k} M \rightarrow T T^{k-1} M$ is an anchor. In local coordinates, as used in [18],

and

$$
\left(x^{i}, y^{(1) j}, \ldots, y^{(k) j}\right) \stackrel{h_{k}}{\rightarrow} y^{(1) j} \frac{\partial}{\partial x^{j}}+2 y^{(2) j} \frac{\partial}{\partial y^{(1) j}}+\cdots+k y^{(k) j} \frac{\partial}{\partial y^{(k-1) j}}
$$

$$
\Gamma=y^{(1) i} \frac{\partial}{\partial x^{i}}+y^{(2) i} \frac{\partial}{\partial y^{(1) i}}+\cdots+k y^{(k) i} \frac{\partial}{\partial y^{(k-1) i}} .
$$

According to [9, pg. 81] it was first considered by Tulczyjew in 1975 . 
Consider a fibered manifold $E \stackrel{\pi}{\rightarrow} M$ and an anchor $\rho: E \rightarrow T M$. We define, $\forall e \in E$,

$$
E_{e}^{\prime}=\left\{v_{e} \in T_{e} E: \pi_{*, e}\left(v_{e}\right)=\rho(e)\right\} \subset T_{e} E
$$

and consider $E^{\prime}=\bigcup_{e \in E} E_{e}^{\prime}$, the canonical projection $\pi^{\prime}: E^{\prime} \rightarrow E$ and the inclusion $\Phi: E^{\prime} \rightarrow T E$.

Proposition 3.1. If $E \stackrel{\pi}{\rightarrow} M$ is a fibered manifold and $E \stackrel{\pi}{\rightarrow} M$ is an anchor, then $E^{\prime} \stackrel{\pi^{\prime}}{\rightarrow} E$ an affine subbundle of $T E$ and $\Phi$ is the inclusion morphism.

Proof. A vector field $X \in \mathcal{X}(E)$ is a section of $E^{\prime}$ iff $\pi_{*, e}\left(X_{e}\right)=\rho(e), \forall e \in E$. Thus $E_{e}^{\prime} \subset T_{e} E$ is an affine subspace of constant dimension, i.e. that of the fiber $E_{e}$.

Notice that assuming that $E \stackrel{\pi}{\rightarrow} M$ is an affine bundle and the local coordinates change on $E$ according to the formulas $\bar{x}^{i}=\bar{x}^{i}\left(x^{j}\right), \bar{y}^{\alpha}=g_{\beta}^{\alpha}\left(x^{j}\right) y^{\beta}+v^{\alpha}\left(x^{j}\right)$, then the change rule of coordinates on fibers of $E^{\prime}$ is given by $\bar{z}^{\beta}\left(x^{i}, y^{\alpha}, z^{\beta}\right)=g_{\gamma}^{\beta}\left(x^{i}\right) z^{\gamma}+\Gamma\left(\bar{y}^{\beta}\right)$.

The above Proposition allows to obtain higher order anchored manifolds $E^{n}, n \geq 1$, starting from an anchor on $E$. We define inductively: $E^{1}=E$ and Proposition 3.1 gives the manifold $E^{n+1}$ and the inclusion $\Phi^{n+1}: E^{n+1} \rightarrow T E^{n}$, using $E^{n}$ and the inclusion $\Phi^{n}: E^{n} \subset T E^{n-1}$ for $n \geq 2$.

In the case when $E=T M$ and the anchor is the identity on fibers, we obtain the construction of $E^{k}=T^{k} M$, as considered at the very beginning of this section.

Let $E \stackrel{\pi}{\rightarrow} M$ be an anchored bundle and $S: E \rightarrow E^{\prime}$ be an affine section, where the affine bundle $E^{\prime} \stackrel{\pi^{\prime}}{\rightarrow} E$ is given by Proposition 3.1. Then $X=\Phi \circ S: E \rightarrow T E$ is a vector field, which is called a semi-spray on $E$. If $S$ has the local form $\left(x^{i}, y^{\alpha}\right) \rightarrow$ $\left(x^{i}, y^{\alpha}, S^{\beta}\left(x^{i}, y^{\alpha}\right)\right)$, then the vector field $X \in \mathcal{X}(E)$ is given locally by $X=\rho^{i}\left(x^{j}, y^{\alpha}\right) \partial / \partial x^{i}$ $+S^{\alpha}\left(x^{i}, y^{\alpha}\right) \partial / \partial y^{\alpha}$.

Let $S$ and $T$ be two semi-sprays and denote by $X=\Phi \circ S, Y=\Phi \circ T: E \rightarrow$ $T E$ the corresponding vector fields. Considering local coordinates, one has $X=\Gamma+$ $S^{\alpha}\left(x^{i}, y^{\beta}\right) \partial / \partial y^{\alpha}$ and $Y=\Gamma+T^{\alpha}\left(x^{i}, y^{\beta}\right) \partial / \partial y^{\alpha}$, where $\Gamma=\rho^{i}\left(x^{j}, y^{\alpha}\right) \partial / \partial x^{i} ;$ thus

$$
[X, Y]=\left(\Gamma\left(T^{\alpha}\right)-\Gamma\left(S^{\alpha}\right)+S^{\beta} \frac{\partial T^{\alpha}}{\partial y^{\beta}}-T^{\beta} \frac{\partial S^{\alpha}}{\partial y^{\beta}}\right) \frac{\partial}{\partial y^{\alpha}}+\left(S^{\alpha}-T^{\alpha}\right)\left[\frac{\partial}{\partial y^{\alpha}}, \Gamma\right] .
$$

Considering the affine bundle $T^{k+1} M \rightarrow T^{k} M$ of the tangent bundle of order $k+1$, we have an affine map $\Phi^{k+1}: T^{k+1} M \rightarrow T T^{k} M$. An affine section $S: T^{k} M \rightarrow$ $T^{k+1} M$ defines a semi-spray of order $k, X \in \mathcal{X}\left(T^{k} M\right)$ by $X=\Phi^{k+1} \circ S$. If $X=$ $\Gamma+S^{i}\left(x^{i}, y^{(1) i}, \ldots, y^{(k) i}\right) \partial / \partial y^{(k) i}$ and $Y=\Gamma+S^{i}\left(x^{i}, y^{(1) i}, \ldots, y^{(k) i}\right) \partial / \partial y^{(k) i}$ are two $k$-semi-sprays, where

$$
\Gamma=y^{(1) i} \frac{\partial}{\partial x^{i}}+y^{(2) i} \frac{\partial}{\partial y^{(1) i}}+\cdots+y^{(k) i} \frac{\partial}{\partial y^{(k-1) i}},
$$

then

$$
[X, Y]=\left(\Gamma\left(T^{i}\right)-\Gamma\left(S^{i}\right)+S^{j} \frac{\partial T^{i}}{\partial y^{(k) j}}-T^{j} \frac{\partial S^{i}}{\partial y^{(k) j}}\right) \frac{\partial}{\partial y^{(k) i}}+\left(S^{i}-T^{i}\right) \frac{\partial}{\partial y^{(k-1) i}},
$$

since $\left[\partial / \partial y^{\alpha}, \Gamma\right]=\partial / \partial y^{(k-1) i}$. Thus the image of the anchor $\Phi^{k+1}$ does not span an involutive distribution. 
A morphism of two anchored bundles $E \stackrel{\pi}{\rightarrow} M$ and $E^{\prime} \stackrel{\pi^{\prime}}{\rightarrow} M^{\prime}$, with anchors $\rho$ and $\rho^{\prime}$ respectively, is a morphism $(f, g)$ of fibered manifolds (i.e. $f: M \rightarrow M^{\prime}$ and $g: E \rightarrow E^{\prime}$ are differentiable maps with $f \circ \pi=\pi^{\prime} \circ g$ ) such that $f_{*} \circ \rho=\rho^{\prime} \circ g$, where $f_{*}$ denotes the differential of $f$.

Proposition 3.2. The differential $g_{*}: T E \rightarrow T E^{\prime}$ restricts to a differentiable map $g^{\prime}: E^{2} \rightarrow E^{\prime 2}$ and the following diagram is commutative:

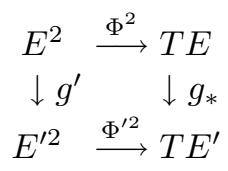

Proof. Consider $e \in E$ and use (3); we have $E_{e}^{2}=\left\{v_{e} \in T_{e} E: \pi_{*, e}\left(v_{e}\right)=\rho(e)\right\} \subset$ $T_{e} E$. Using also the morphism conditions, we have $\pi_{*, g(e)}^{\prime}\left(g_{*, e}\left(v_{e}\right)\right)=\left(\pi^{\prime} \circ g\right)_{*, e}\left(v_{e}\right)=$ $(f \circ \pi)_{*, e}\left(v_{e}\right)=f_{*, \pi(e)}\left(\pi_{*, e}\left(v_{e}\right)\right)=f_{*, \pi(e)}(\rho(e))=\rho^{\prime}\left(g(e)\right.$, thus $g_{*, e}\left(v_{e}\right) \in E_{g(e)}^{\prime(2)}$.

Notice that commutativity of the diagram says that $\left(g, g^{\prime}\right)$ is a morphism of anchored bundles $E^{2}$ and $E^{\prime 2}$.

Using this Proposition, we obtain the following statement.

Proposition 3.3. For $k \geq 2$ there is a morphism of anchored bundles $\left(g^{k-1}, g^{k}\right)$, from $E^{k}$ to $E^{\prime k}$, such that $g^{0}=f$ and $g^{1}=g$, i.e. the diagram

$$
\begin{array}{cc}
E^{k} & \stackrel{\Phi^{k}}{\longrightarrow} T E^{k-1} \\
\downarrow g^{k} & \downarrow g_{*}^{k-1} \\
E^{\prime k} & \stackrel{\Phi^{\prime k}}{\longrightarrow} T E^{\prime k-1}
\end{array}
$$

is commutative.

In particular, the anchor $\rho: E \rightarrow T M$ is a morphism of anchored bundles, thus we obtain the following statement.

Proposition 3.4. For every $k \geq 2$ there is a morphism of anchored bundles $\left(\rho^{k-1}, \rho^{k}\right)$, from $E^{k}$ to $T^{k} M$, such that the diagram

$$
\begin{array}{cc}
E^{k} & \stackrel{\tilde{\Phi}^{k}}{\longrightarrow} T E^{k} \\
\downarrow \rho^{k+1} & \downarrow \rho_{*}^{k} \\
T^{k} M & \stackrel{\Phi^{k}}{\longrightarrow} T T^{k} M
\end{array}
$$

is commutative.

There is a canonical morphism of vector bundles over $E^{k-1}, T^{*} E^{k-1} \stackrel{\pi_{1}}{\rightarrow} E^{k *}=$ $E^{k-1} \times_{M} \bar{E}^{*}$. Using local coordinates, $\left(x^{i}, y^{(1) \alpha}, \ldots, y^{(k-1) \alpha}, p_{(0) i}, p_{(a) \alpha}, \ldots, p_{(k-1) \alpha}\right) \stackrel{\pi_{1}}{\rightarrow}$ $\left(x^{i}, \ldots, y^{(1) \alpha}, \ldots, y^{(k-1) \alpha}, p_{(k-1) \alpha}\right)$; it is a surjection on each fiber and also a canonical projection of an affine bundle.

Let $L: \tilde{E}^{k} \rightarrow \mathbb{R}$ be a hyperregular lagrangian of order $k$ on the anchored affine bundle $E$, where $\tilde{E}^{k}$ is an open fibered submanifold of $E^{k}$, and $h$ be the affine hamiltonian 
given by Theorem (2.1), using Legendre transformation. Then the formula

$$
\begin{aligned}
\mathcal{E}= & p_{(0) i} \rho^{i}\left(x^{j}, y^{(1) \alpha}\right)+p_{(1) \alpha} y^{(2) \alpha}+\cdots+p_{(k-2) \alpha} y^{(k-1) \alpha} \\
& +H_{0}\left(x^{i}, y^{(1) \alpha}, \ldots, y^{(k-1) \alpha}, p_{(k-1) \alpha}\right)
\end{aligned}
$$

defines a global function $\mathcal{E}: \check{E}^{k-1}=\pi_{1}^{-1}\left(\mathcal{L}\left(\tilde{E}^{k}\right)\right) \rightarrow \mathbb{R}$, where $H_{0}$ are the local functions defined by the affine hamiltonian $h$. We call $\mathcal{E}$ the energy of $L$ (or $h$ ). Then $\mathcal{E}$ is a singular hamiltonian on the manifold $E^{k-1}$ and there is a Legendre* fibered manifold on each fiber. We denote below by $\mathcal{H}$ and $\mathcal{L}$ the Legendre* map of $\mathcal{E}$ and the Legendre map of $L$ respectively.

THEOREM 3.1. Let $s: \mathcal{L}\left(\tilde{E}^{k}\right) \rightarrow \check{E}^{k-1}$ be a section of the fibered submanifold $\check{E}^{k-1} \rightarrow$ $\mathcal{L}\left(\tilde{E}^{k}\right)$. Then the following statements hold:

1. The image of $s$ is transverse on every fiber to the fibers of the Legendre* fibration, giving a Legendre triple $\left(\mathcal{H}\left(\check{E}^{k-1}\right)=\tilde{E}^{k},\left(\mathcal{H}_{\mid s\left(\mathcal{L}\left(\tilde{E}^{k}\right)\right)}\right)^{-1}=s \circ \mathcal{L}, s\left(\mathcal{L}\left(\tilde{E}^{k}\right)\right)\right)$.

2. The Legendre triple defined above is linearizable and $\mathcal{E}$ is its hamiltonian.

Proof. Using local coordinates, the local forms of the Legendre* fibration of the fiber and the section $s$ are:

$$
\begin{aligned}
&\left(x^{i}, y^{(1) \alpha}, \ldots, y^{(k-1) \alpha},\left(p_{(0) i}, p_{(1) \alpha}, \ldots, p_{(k-1) \alpha}\right)\right) \rightarrow\left(x^{i}, y^{(1) \alpha}, \ldots, y^{(k-1) \alpha}\right. \\
&\left.\left(\rho^{i}\left(x^{j}, y^{(1) \alpha}\right), y^{(2) \alpha}, \ldots, y^{(k-1) \alpha}, \frac{\partial H_{0}}{\partial p_{\alpha}}\left(x^{i}, y^{(1) \alpha}, \ldots, y^{(k-1) \alpha}, p_{(k-1) \alpha}\right)\right)\right)
\end{aligned}
$$

and

$$
\left(x^{i}, y^{(1) \alpha}, \ldots, y^{(k-1) \alpha},\left(p_{\alpha}\right)\right) \stackrel{s}{\rightarrow}\left(x^{i}, y^{(1) \alpha}, \ldots, y^{(k-1) \alpha},\left(s_{(0) i}, s_{(1) \alpha}, \ldots, s_{(k-2) \alpha}, p_{\alpha}\right)\right)
$$

respectively, where the coordinates of $z \in \tilde{E}^{k-1}$ are $\left(x^{i}, y^{(1) \alpha}, \ldots, y^{(k-1) \alpha}\right)$, the local functions $s_{(1) \alpha}, \ldots, s_{(k-2) \alpha}$ have as variables $\left(x^{i}, y^{(1) \alpha}, \ldots, y^{(k-1) \alpha}, p_{\alpha}\right)$ and the inside parentheses denote coordinates on fibers. Thus $\mathcal{H}_{z}\left(\check{E}_{z}^{k-1}\right)=E_{z}^{k} \subset T_{z} \tilde{E}^{k-1}$ and $s_{z}\left(\mathcal{L}_{z}\left(\tilde{E}_{z}^{k}\right)\right) \subset \check{E}_{z}^{k-1}$ have as coordinates $\left(y^{(k) \alpha}\right) \rightarrow\left(\rho^{i}\left(x^{j}, y^{(1) \alpha}\right), y^{(2) \alpha}, \ldots, y^{(k-1) \alpha}, y^{(k) \alpha}\right)$ and $\left(p_{\alpha}\right) \rightarrow\left(s_{(0) i}, s_{(1) \alpha}, \ldots, s_{(k-2) \alpha}, p_{\alpha}\right)$ respectively. Using these coordinates, the local form of $\left(\mathcal{H}_{\mid s\left(\mathcal{L}\left(\tilde{E}^{k}\right)\right)}\right)^{-1}$ is the same as that of $s \circ \mathcal{L}$, i.e.

$$
\begin{aligned}
\left(x^{i}, y^{(1) \alpha}, \ldots,\right. & \left.y^{(k) \alpha}\right) \leftrightarrow\left(x^{i}, y^{(1) \alpha}, \ldots, y^{(k-1) \alpha}, y^{(1) \alpha}, \ldots, y^{(k) \alpha}\right) \\
& \rightarrow\left(s_{(0) i}\left(x^{i}, y^{(1) \alpha}, \ldots, y^{(k-1) \alpha}, \frac{\partial L}{\partial y^{(k) \alpha}}\right), s_{(1) \alpha}, \ldots, s_{(k-2) \alpha}, \frac{\partial L}{\partial y^{(k) \alpha}}\right) .
\end{aligned}
$$

Thus the first assertion follows.

Let $\left(X^{(0) i}, X^{(1) \alpha}, \ldots, X^{(k-1) \alpha}\right)$ and $\left(\rho^{i}\left(x^{i}, y^{(1) \alpha}\right), y^{(2) \alpha}, \ldots, y^{(k-1) \alpha}, y^{(k) \alpha}\right)$ be the coordinates on fibers in $z$ of a point $w \in T_{z} \tilde{E}^{k-1}$, respectively $w_{0} \in \tilde{E}_{z}^{k} \subset T_{z} \tilde{E}^{k-1}$, where $z$ has coordinates $\left(x^{i}, y^{(1) \alpha}, \ldots, y^{(k-1) \alpha}\right)$. The coordinates considered below are ones on fibers.

Considering the correspondence $\tilde{E}_{z}^{k} \stackrel{\mathcal{L}}{\rightarrow} T_{z}^{*} \tilde{E}^{k-1}$, i.e.

$$
\begin{aligned}
\left(y^{(k) \alpha}\right) \leftrightarrow\left(\rho^{i}\left(x^{i}, y^{(1) \alpha}\right), y^{(2) \alpha}, \ldots, y^{(k-1) \alpha}, y^{(k) \alpha}\right) & \\
& \stackrel{\mathcal{L}_{z}}{\longrightarrow}\left(s_{(0) i}, s_{(1) \alpha}, \ldots, s_{(k-2) \alpha}, \frac{\partial L}{\partial y^{(k) \alpha}}\left(x^{i}, y^{(1) \alpha}, \ldots, y^{(k-1) \alpha}, y^{(k) \alpha}\right)\right),
\end{aligned}
$$


the condition that $w-w_{0}$ belongs to the polar of the subspace tangent to $\mathcal{L}_{z}\left(\tilde{E}_{z}^{k}\right)$ at a point of coordinates $\left(\rho^{i}\left(x^{i}, y^{(1) \alpha}\right), y^{(2) \alpha}, \ldots, y^{(k-1) \alpha}, y^{(k) \alpha}\right)$ is:

$$
\begin{aligned}
\left(X^{(0) i}-\rho^{i}\left(x^{j}, y^{(1) \gamma}\right)\right) \frac{\partial s_{(0) i}}{\partial p_{\beta}} \frac{\partial^{2} L}{\partial y^{\beta} \partial y^{\alpha}}+\left(X^{(1) \gamma}-y^{(2) \gamma}\right) \frac{\partial s_{(0) \gamma}}{\partial p_{\beta}} \frac{\partial^{2} L}{\partial y^{\beta} \partial y^{\alpha}} \\
\quad+\cdots+\left(X^{(k-2) \gamma}-y^{(k-2) \gamma}\right) \frac{\partial s_{(0) \gamma}}{\partial p_{\beta}} \frac{\partial^{2} L}{\partial y^{\beta} \partial y^{\alpha}}+\left(X^{(k-1) \gamma}-y^{(k-1) \gamma}\right) \frac{\partial^{2} L}{\partial y^{\gamma} \partial y^{\alpha}}=0
\end{aligned}
$$

thus

$$
\begin{aligned}
\left(X^{(0) i}-\rho^{i}\left(x^{j}, y^{(1) \gamma}\right)\right) \frac{\partial s_{(0) i}}{\partial p_{\beta}}+ & \left(X^{(1) \gamma}-y^{(2) \gamma}\right) \frac{\partial s_{(0) \gamma}}{\partial p_{\beta}} \\
& +\cdots+\left(X^{(k-2) \gamma}-y^{(k-1) \gamma}\right) \frac{\partial s_{(0) \gamma}}{\partial p_{\beta}}+\left(X^{(k-1) \beta}-y^{(k) \beta}\right)=0 .
\end{aligned}
$$

It follows that

$$
\begin{aligned}
X^{(k-1) \beta}= & y^{(k) \beta}+\left(\rho^{i}\left(x^{j}, y^{(1) \gamma}\right)-X^{(0) i}\right) \frac{\partial s_{(0) i}}{\partial p_{\beta}}+\left(y^{(2) \gamma}-X^{(1) \gamma}\right) \frac{\partial s_{(0) \gamma}}{\partial p_{\beta}}+\cdots \\
& +\left(y^{(k-1) \gamma}-X^{(k-2) \gamma}\right) \frac{\partial s_{(0) \gamma}}{\partial p_{\beta}}
\end{aligned}
$$

Considering all $w_{0} \in \tilde{E}_{z}^{k}$ and $w$ as above, then $w$ generate the total space of an affine bundle $\xi_{\tilde{E}_{z}^{k}}$ with projection $w \rightarrow w_{0}$. The zero section of this affine bundle is obtained considering $w-w_{0}=\overline{0}$.

Let $w_{0}^{\prime} \in \tilde{E}_{z}^{k}$ have coordinates $\left(\rho^{i}\left(x^{i}, y^{(1) \alpha}\right), y^{(2) \alpha}, \ldots, y^{(k-1) \alpha}, y_{0}^{(k) \alpha}\right)$. For $w_{0}$ in a small enough open neighborhood $U_{w_{0}^{\prime}}$ of $w_{0}^{\prime}$ in $\tilde{E}_{z}^{k}$ the map $\left(X^{(0) i}, X^{(1) \alpha}, \ldots, X^{(k-2) \alpha}\right.$, $\left.y^{(k) \alpha}\right) \rightarrow\left(X^{(0) i}, X^{(1) \alpha}, \ldots, X^{(k-2) \alpha}, X^{(k-1) \beta}\right)$, where $X^{(k-1) \alpha}$ is given by $(5)$, is a local diffeomorphism of two small neighborhoods of $\left(\rho^{i}\left(x^{j}, y^{(1) \gamma}\right), y^{(2) \alpha}, \ldots, y^{(k) \alpha}\right)$. We can consider $\left(X^{(0) i}, X^{(1) \alpha}, \ldots, X^{(k-2) \alpha}, y^{(k) \alpha}\right)$ as local coordinates on $\xi_{\tilde{E}_{z}^{k}}$ (as a differentiable manifold) and $\left(X^{(0) i}, X^{(1) \alpha}, \ldots, X^{(k-2) \alpha}, X^{(k-1) \beta}\right)$ as coordinates in the vector space $T_{z} \tilde{E}^{k-1}$, representing the same points of an open subset $V_{w_{0}^{\prime}} \subset T_{z} \tilde{E}^{k-1} \cap \xi_{\tilde{E}_{z}^{k}}$. The union $A_{z}=\bigcup_{w_{0}^{\prime} \in \tilde{E}_{z}^{k}} V_{w_{0}^{\prime}}$ gives an open neighborhood of $\tilde{E}_{z}^{k}$ in $T_{z} \tilde{E}^{k-1} \cap \xi_{\tilde{E}_{z}^{k}}$ (where we can identify points in $T_{z} \tilde{E}^{k-1}$ with points in $\left.\xi_{\tilde{E}_{z}^{k}}\right)$. It follows that the Legendre triple is $T \tilde{E}^{k-1}$-linearizable.

We prove in what follows that the Legendre triple is $T^{*} \tilde{E}^{k-1}$-linearizable.

Let us consider $\left(p_{(0) i}, p_{(1) \alpha}, \ldots, p_{(k-1) \alpha}\right)$ and $\left(s_{(0) i}, s_{(1) \alpha}, \ldots, s_{(k-2) \alpha}, p_{\alpha}\right)$ as coordinates on fibers in $z$ of a point $\Omega \in T_{z}^{*} \tilde{E}^{k-1}$, respectively $\Omega_{0} \in s_{z}\left(\mathcal{L}_{z}\left(\tilde{E}_{z}^{k}\right)\right) \subset T_{z}^{*} \tilde{E}^{k-1}$, where $z \in \tilde{E}^{k-1}$ has coordinates $\left(x^{i}, y^{(1) \alpha}, \ldots, y^{(k-1) \alpha}\right)$ and $s_{(0) i}, s_{(1) \alpha}, \ldots, s_{(k-2) \alpha}$ are as in the first part of the proof.

Considering the correspondence

$$
\begin{aligned}
\left(p_{\alpha}\right) \leftrightarrow & \left(s_{(0) i}, s_{(1) \alpha}, \ldots, s_{(k-2) \alpha}, p_{\alpha}\right) \\
& \stackrel{\mathcal{H}_{\mid s\left(\mathcal{L}\left(\tilde{E}^{k}\right)\right)}}{\longrightarrow}\left(\rho^{i}\left(x^{j}, y^{(1) \alpha}\right), y^{(2) \alpha}, \ldots, y^{(k-1) \alpha}, \frac{\partial H_{0}}{\partial p_{\alpha}}\left(x^{i}, y^{(1) \alpha}, \ldots, y^{(k-1) \alpha}, p_{(k-1) \alpha}\right)\right),
\end{aligned}
$$

the condition that $\Omega-\Omega_{0}$ belongs to the polar of the subspace tangent to $\tilde{E}_{z}^{k}$ at a point 
of coordinates $\left(\rho^{i}\left(x^{i}, y^{(1) \alpha}\right), y^{(2) \alpha}, \ldots, y^{(k-1) \alpha}, y^{(k) \alpha}\right)$ is

$$
\left.\left(p_{(k-1) \alpha}-p_{\alpha}\right) \frac{\partial^{2} H_{0}}{\partial p_{\alpha} \partial p_{\beta}}\left(x^{i}, y^{(1) \alpha}, \ldots, y^{(k-1) \alpha}, p_{(k-1) \alpha}\right)\right)=0,
$$

thus $p_{(k-1) \alpha}=p_{\alpha}$.

Let us consider $\eta_{s_{z}\left(\mathcal{L}_{z}\left(\tilde{E}_{z}^{k}\right)\right)}=\pi_{1}^{-1}\left(\mathcal{L}_{z}\left(\tilde{E}_{z}^{k}\right)\right) \subset T_{z}^{*} \tilde{E}^{k-1}$ and a projection of an affine bundle $\eta_{s_{z}\left(\mathcal{L}_{z}\left(\tilde{E}_{z}^{k}\right)\right)} \rightarrow s_{z}\left(\mathcal{L}_{z}\left(\tilde{E}_{z}^{k}\right)\right)$ given by

$$
\left(p_{(0) i}, p_{(1) \alpha}, \ldots, p_{(k-1) \alpha}\right) \rightarrow\left(s_{(0) i}, s_{(1) \alpha}, \ldots, s_{(k-2) \alpha}, p_{(k-1) \alpha}\right),
$$

where $s_{(0) i}, s_{(1) \alpha}, \ldots, s_{(k-2) \alpha}$ have as variables $\left(x^{i}, y^{(1) \alpha}, \ldots, y^{(k-1) \alpha}, p_{(k-1) \alpha}\right)$. But $\mathcal{L}_{z}\left(\tilde{E}_{z}^{k}\right) \subset \bar{E}_{z}^{k *}$ is an open subset (since $\mathcal{L}\left(\tilde{E}^{k}\right) \subset \bar{E}^{k *}$ is an open fibered submanifold), thus $\eta_{s_{z}\left(\mathcal{L}_{z}\left(\tilde{E}_{z}^{k}\right)\right)} \subset T_{z}^{*} \tilde{E}^{k-1}$ is an open subset. It follows that the Legendre triple is $T^{*} \tilde{E}^{k-1}$-linearizable. A straightforward computation shows that the local form of the hamiltonian given using Proposition 1.6 is just $\mathcal{E}$.

In the case when the anchor bundle $E \stackrel{\pi}{\rightarrow} M$ is the tangent space $T M \rightarrow M$, then there is a section $s$ that depends only on the lagrangian $L$. It is given by the local formula

$$
\begin{aligned}
& \left(x^{i}, y^{(1) i}, \ldots, y^{(k-1) i}, p_{(k-1) i}\right) \\
& \quad \stackrel{s_{1}}{\rightarrow}\left(x^{i}, y^{(1) i}, \ldots, y^{(k-1) i}, \frac{\partial L}{\partial y^{(1) i}}\left(x^{i}, y^{(1) i}, \ldots, y^{(k-1) i}, h^{i}\right), \ldots,(k-1) \frac{\partial L}{\partial y^{(k-1) i}}, p_{(k-1) i}\right),
\end{aligned}
$$

where $h^{i}=\left(\partial H_{0} / \partial p_{i}\right)\left(x^{i}, y^{(1) i}, \ldots, y^{(k-1) i}, p_{i}\right)$ is given by the inverse of the Legendre transformation of $L$ (i.e. $\left.\left(\partial L / \partial y^{(k) i}\right)\left(x^{i}, y^{(1) i}, \ldots, y^{(k-1) i}, h^{i}\right)=p_{i}\right)$.

\section{References}

[1] V. I. Arnold and A. B. Givental, Symplectic Geometry, in: Dinamical Systems IV, Encyclopedia of Mathematical Sciences, Springer-Verlag, 1990.

[2] M. N. Boyom, Anchored vector bundles and algebroids, arXiv:math.dg/0208012.

[3] F. Cantrijn and B. Langerock, Generalised connections over a vector bundle map, Diff. Geom. Appl. 18 (2003), 295-317.

[4] K. Grabowska, J. Grabowski and P. Urbański, Frame independent mechanics: geometry on affine bundles, arXiv:math.dg/0510069.

[5] K. Grabowska, J. Grabowski and P. Urbański, Lie brackets on affine bundles, Ann. Global Anal. Geom. 24 (2003), 101-130.

[6] J. Grabowski and P. Urbański, Algebroids-general differential calculi on vector bundles, J. Geom. Phys. 31 (1999), 111-141.

[7] D. Iglesias, J. C. Marrero, E. Padron and D. Sosa, Lagrangian submanifolds and dynamics on Lie algebroids, arXiv:math.DG/0505117.

[8] B. Langerock, Leafwise holonomy of connections over a bundle map, Differ. Geom. Appl. 18 (2003), 295-317.

[9] M. de Leon and P. R. Rodrigues, Generalized Classical Mechanics and Field Theory, North-Holland, 1985. 
[10] M. de Leon, J. Marrero and E. Martinez, Lagrangian submanifolds and dynamics on Lie algebroids, arXiv:math.dg/0407528

[11] P. Libermann, Lie algebroids and mechanics, Archivum Mathematicum 32 (1996), 147162.

[12] K. Mackenzie, Lie Groupoids and Lie Algebroids in Differential Geometry, London Mathematical Society Lecture Note Series 124, Cambridge Univ. Press, 1987.

[13] J. C. Marrero, D. Martin De Diego and E. Martinez, Discrete Lagrangian and Hamiltonian mechanics on Lie groupoids, arXiv:math.DG/0506299.

[14] J. Marsden and T. Ratiu, Introduction to Mechanics and Symmetry, 2nd ed., SpringerVerlag, New York, 1999.

[15] E. Martinez, Classical field theory on Lie algebroids: multisymplectic formalism, arXiv:math.DG/0411352.

[16] E. Martinez, T. Mestdag and W. Sarlet, Lie algebroid structures and Lagrangian systems on affine bundles, J. Geom. Phys. 44 (2002), 70-95.

[17] R. Miron, Sur la géométrie des espaces d'Hamilton, C.R. Acad Sci. Paris 306 (1988), 195-198.

[18] R. Miron, The Geometry of Higher Order Lagrange Spaces. Applications to Mechanics and Physics, Kluwer, Dordrecht, 1997.

[19] R. Miron, The Geometry of Higher-Order Hamilton Spaces. Applications to Hamiltonian Mechanics, Kluwer, Dordrecht, 2003.

[20] R. Miron and Gh. Atanasiu, Differential geometry of the k-osculator bundle, Rev. Roum. Math. Pures Appl. 41 (1996), 205-236.

[21] R. Miron, Hamilton spaces of order k, Int. J. Theor. Phys. 39 (2000), 2327-2336.

[22] M. Popescu, On induced non-linear connections, An. Univ. Al. I. Cuza Iaşi, seria mat. 42 (1996), 119-126.

[23] M. Popescu, Semisprays induced on submanifolds, in: New Developments in Diff. Geom., Kluwer Academic Publ., 1998, 317-328.

[24] P. Popescu, Categories of modules with differentials, Journal of Algebra 185 (1996), 50-73.

[25] P. Popescu and M. Popescu, On Hamiltonian submanifolds (I), Balkan Journal of Differential Geometry and its Applications 7 (2002), no. 2, 79-86.

[26] P. Popescu and M. Popescu, A general background of higher order geometry and induced objects on subspaces, Balkan Journal of Differential Geometry and its Applications 7 (2002), no. 1, 79-90.

[27] M. Puta, Hamiltonian Mechanical Systems and Geometric Quantization, Kluwer Acad. Publ., 1993.

[28] W. M. Tulczyjew and P. Urbański, A slow and careful Legendre transformation for singular Lagrangians, arXiv:math-ph/9909029.

[29] P. Urbański, An affine framewark for analytical mechanics, in: Classical and Quantum Integrability, Banach Center Publications 59, Warszawa, 2003, 257-279.

[30] A. Weinstein, Lagrangian mechanics and groupoids, in: Proceedings of Mechanics Day, Fields Institute. 
